\title{
Particle-in-cell and Weak Turbulence Simulations of Plasma Emission
}

\author{
Sang-Yun Lee ${ }^{1}\left(10\right.$, L. F. Ziebell ${ }^{2}$ (1) , P. H. Yoon ${ }^{1,3,4}$ (1), R. Gaelzer ${ }^{2}$ (1), and E. S. Lee ${ }^{1}$ \\ ${ }^{1}$ School of Space Research, Kyung Hee University, Yongin, Gyeonggi 17104, Republic of Korea; lizagd@khu.ac.kr, yoonp@umd.edu, eslee@khu.ac.kr \\ ${ }_{3}^{2}$ Instituto de Física, UFRGS, 91501-970 Porto Alegre, RS, Brazil; luiz.ziebell@ufrgs.br, rudi.gaelzer@ufrgs.br \\ ${ }^{3}$ Institute for Physical Science and Technology, University of Maryland, College Park, MD 20742-2431, USA \\ ${ }^{4}$ Korea Astronomy and Space Science Institute, Daejeon 34055, Republic of Korea \\ Received 2018 November 4; revised 2018 November 18; accepted 2018 November 26; published 2019 January 23
}

\begin{abstract}
The plasma emission process, which is the mechanism for solar type II and type III radio burst phenomena, is studied by means of particle-in-cell (PIC) and weak turbulence (WT) simulation methods. "Plasma emission" is meant as a loose description of a series of processes, starting from the solar flare-associated electron beam exciting Langmuir and ion-acoustic turbulence, and subsequent partial conversion of beam energy into radiation energy by nonlinear processes. PIC simulation is rigorous but the method is computationally intense, and it is difficult to diagnose the results. The numerical solution of equations of WT theory, known as WT simulation, on the other hand, is efficient and naturally lends itself to diagnostics because various terms in the equation can be turned on or off. Nevertheless, WT theory is based upon a number of assumptions. It is therefore desirable to compare the two methods, which we do for the first time with numerical solutions of the complete set of equations of WT theory and a two-dimensional electromagnetic PIC simulation. Upon making quantitative comparisons, it is found that WT theory is largely valid, although some discrepancies are also found. The present study also indicates that large computational resources are required in order to accurately simulate the radiation emission processes, especially for low electron beam speeds, such that it may be more advantageous to employ the WT method in order to describe the radiation emission itself. Findings from the present paper thus imply that both methods may be useful for the study of solar radio emissions, as they are complementary.
\end{abstract}

Key words: methods: analytical - methods: numerical - plasmas - radiation processes: thermal - turbulence waves

\section{Introduction}

Solar radio burst phenomena at meter wavelengths were discovered and subsequently classified into five categories, beginning in the 1950s (Wild \& McCready 1950; Wild 1950a, 1950b; Wild et al. 1954). The phenomena are still being investigated to this day. The present work relates particularly to type II and type III radio bursts. Both phenomena are related to high-energy beams of electrons produced during solar flares and coronal mass ejections, which occur in the solar chromosphere and corona (Benz 2017), and are characterized by emissions in the VHF band, with frequencies ranging from $10 \mathrm{MHz}$ to approximately $300 \mathrm{MHz}$ (hence with wavelengths of the order of meters). Dynamic spectra of both types II and III typically display negative frequency drift with time, but while type III bursts typically occur in short bursts lasting a few minutes, type II are characterized by a much slower downward drift rate that can last for several tens of minutes (Bastian et al. 1998; Ergun et al. 1998; Cane et al. 2002; Reiner et al. 2009; Hudson 2011; Reid \& Ratcliffe 2014).

The generally accepted mechanism by which type II and III solar radio bursts are generated was initially proposed by the pioneering work of Ginzburg \& Zheleznyakov (1958) and has been greatly improved by several contributions since then. According to the standard theory, disruptive events occurring in the solar chromosphere, such as solar flares, create bursts of energetic electrons that stream outward from the Sun, along open magnetic field lines. As these bursts propagate through the corona, which already contains a denser population of particles in a quasithermalized state, a beam-plasma instability is triggered when the speed of the beam electrons exceeds the thermal velocity of the background electron population. This bump-on-tail instability then excites the exponential growth of dispersive Langmuir waves
( $L$ mode waves), starting from the initially low level of local thermal radiation fields, which are predominantly emitted in the same direction as the beam. As the Langmuir waves are convectively amplified along their ray paths, the wave intensity becomes sufficiently high for nonlinear processes to start taking place. According to the weak turbulence (WT) theory of plasmas, the most important nonlinear processes in this phase are the threewave decay/coalescence and nonlinear wave-particle scattering, which will first amplify the backscattered Langmuir mode from the thermal background, mostly due to the coupling of the forward-propagating $L$ mode with the ion-acoustic $(S)$ mode. At later stages of the nonlinear turbulent processes, when the backward $L$ mode reaches a sufficiently high intensity level, subsequent wave-wave and wave-particle interactions promote its coupling with the forward $L$ mode, thereby exciting the electromagnetic (transverse, $T$ ) mode. Longer-timescale processes can lead to the excitation of harmonics of the transverse mode. The entire series of events, which was broadly described above, is known in the literature as the plasma emission process and is documented in the literature (Tsytovich 1967; Kaplan \& Tsytovich 1968; Goldman \& DuBois 1982; Melrose 1982, 1987; Goldman 1983; Cairns 1987a; Robinson \& Cairns 1998a, 1998b; Zlotnik et al. 1998; Kontar 2001a, 2001b, 2001c; Gosling et al. 2003; Li et al. 2005a, 2005b, 2006, 2008, 2011, 2012; Ratcliffe et al. 2012; Krafft et al. 2013; Reid \& Kontar 2017).

The plasma emission mechanism has also been studied within the framework of the generalized weak turbulence (GWT) theory (Yoon 2000, 2005, 2006; Yoon et al. 2012b). In this theory, the evolution of the turbulence level in a thermal plasma is described by a set of kinetic equations for the velocity distribution functions (VDFs) of the plasma species 
and for the spectral wave intensities of the various normal modes interacting with the particles. Examples of applications of GWT theory to the study of the nonlinear evolution of the beam-plasma instability, taking into account wave-wave and nonlinear wave-particle interactions, can be found in Ziebell et al. (2001, 2008b), Gaelzer et al. (2008), Ziebell et al. (2008a, 2011a, 2011b, 2012), Yoon et al. (2012a), and Ziebell et al. (2014c, 2014a, 2014b, 2015, 2016). In particular, in Ziebell et al. (2014b, 2015) all the steps involved in the plasma emission process are considered. In addition to the wave kinetic equations for the forward/backward $L$ and $S$ modes, containing all the usual linear and nonlinear interactions included in the traditional WT theory, the above-mentioned publications also included the kinetic equations for the forward/backward transverse modes. The full set of kinetic equations for particles and waves is self-consistently solved during a sufficiently long evolution time that shows not only the excitation of the fundamental $T$ mode, but the first two transverse harmonic modes as well. The results obtained by Ziebell et al. (2014b, 2015) give additional support to the hypothesis that the underlying generating mechanism of Type II/III solar radio bursts is the plasma emission process.

On another theoretical front, some authors have employed particle-in-cell (PIC) simulation technique to the question of plasma emission as the mechanism behind solar radio emissions. Some examples of this strain of research are Kasaba et al. (2001), Karlický \& Vandas (2007), Rhee et al. (2009a, 2009b), Umeda (2010), Ganse et al. (2012a, 2012b), Thurgood \& Tsiklauri (2015), and Henri et al. (2019).

Until now, the few attempts that have been made to directly compare, either qualitatively or quantitatively, the results obtained from WT theory and from numerical simulations have all been of a limited scope. Rha et al. (2013) employed PIC simulation to test a theory of the generation of asymmetric superthermal tails in the electronic distribution function. According to the theory (Yoon et al. 2012a), asymmetric tails can be created due to nonlinear interactions of electrons with $L$ and $S$ waves, when the ion-electron temperature ratio $\left(T_{i} / T_{e}\right)$ varies within the range $0.1 \lesssim T_{i} / T_{e} \lesssim 1$. Ziebell et al. (2014c) employed a simulation to test a theory for the generation of a quasi-thermal electromagnetic radiation field, which was a result of the time-asymptotic state of the nonlinear interaction of the transverse mode with the longitudinal modes and particles, in the absence of free energy sources (e.g., beams) and collisions (i.e., no Brehmsstrahlung emission). On the other hand, Ratcliffe et al. (2014) performed a direct comparison of the plasma emission mechanism with a PIC simulation. However, the WT formulation that was tested was onedimensional and the Langmuir wave kinetic equation contained only the three-wave decay term, besides the usual quasi-linear diffusion term. Moreover, the transverse mode kinetic equation was absent in their comparison.

Here, we report for the first time a detailed comparison between full two-dimensional (2D) plasma emission theory and a 2D PIC simulation. The complete numerical solutions of $2 \mathrm{D}$ equations of GWT theory can be referred to as the WT simulation, and they are directly compared to the numerical results obtained from the 2D PIC code simulation. Employing the same sets of physical parameters, the plasma emission process is simulated with both the $2 \mathrm{D}$ relativistic electromagnetic (EM) PIC simulation, employing a physical proton-to-electron mass ratio, and the GWT theory, employing the full set of 2D self-consistent kinetic equations for the particles and for $L, S$, and $T$ waves. The results show a reasonably good quantitative agreement between the results from both approaches, with better agreement obtained for the low-beam speed regime.

This paper is organized as follows. In Section 2, a short overview of GWT theory, as well as a concise description of the PIC code, are presented. In Section 3 the results from both approaches are presented and the comparison between them is carried out. Finally, in Section 4 we summarize our major findings and present our conclusions.

\section{WT versus PIC Simulations}

We begin the present section with a brief overview of the numerical approach to solving the complete set of equations for electromagnetic WT theory, which is designated the "weak turbulence simulation." The essential theoretical developments for GWT theory that are relevant to the study of plasma emission can be found in Yoon (2006), Yoon et al. (2012b), and Ziebell et al. (2015). The theory describes weakly turbulent nonlinear interactions of Langmuir $(L)$, ion-sound $(S)$, and transverse electromagnetic $(T)$ waves, as well as the electrons and protons. The longitudinal electric field wave energy density is given in spectral form by the sum of Langmuir and ion-sound mode intensities, and is defined by

$$
\left\langle\delta E_{\|}^{2}\right\rangle_{\boldsymbol{k}, \omega}=\sum_{\sigma= \pm 1} \sum_{\alpha=L, S} I_{\alpha}^{\sigma}(\boldsymbol{k}) \delta\left(\omega-\sigma \omega_{\boldsymbol{k}}^{\alpha}\right)
$$

where $I_{\alpha}^{\sigma}(\boldsymbol{k})$ represents the individual mode intensity, $\sigma= \pm 1$ represents the forward/backward propagation direction of wave modes defined with respect to the electron beam propagation direction, and $\omega_{\boldsymbol{k}}^{\alpha}$ denotes the wave dispersion relation. The electric and magnetic field wave energy densities for the transverse mode $T$ are expressed in terms of the $T$ mode wave intensity $I_{T}^{\sigma}(\boldsymbol{k})$ by

$$
\begin{aligned}
\left\langle\delta E_{\perp}^{2}\right\rangle_{\boldsymbol{k} \omega} & =\sum_{\sigma= \pm 1} I_{T}^{\sigma}(\boldsymbol{k}) \delta\left(\omega-\sigma \omega_{\boldsymbol{k}}^{T}\right), \\
\left\langle\delta B^{2}\right\rangle_{\boldsymbol{k} \omega} & =\sum_{\sigma= \pm 1}\left|\frac{c k}{\omega_{k}^{T}}\right|^{2} I_{T}^{\sigma}(\boldsymbol{k}) \delta\left(\omega-\sigma \omega_{\boldsymbol{k}}^{T}\right) .
\end{aligned}
$$

In the above $\sigma= \pm 1$ represents the directions of the wave phase speed with respect to the initial electron beam propagation direction, with $\sigma=+1$ representing the forward propagation, and $\sigma=-1$ denoting the backward direction. The linear dispersion relations for $L, S$, and $T$ modes are given, respectively, by

$$
\begin{aligned}
\omega_{\boldsymbol{k}}^{L} & =\omega_{p e}\left(1+\frac{3}{2} k^{2} \lambda_{D}^{2}\right), \\
\omega_{\boldsymbol{k}}^{S} & =\frac{k c_{S}\left(1+3 T_{e} / T_{i}\right)^{1 / 2}}{\left(1+k^{2} \lambda_{D}^{2}\right)^{1 / 2}}, \\
\omega_{\boldsymbol{k}}^{T} & =\left(\omega_{p e}^{2}+c^{2} k^{2}\right)^{1 / 2},
\end{aligned}
$$


where

$$
\begin{aligned}
& \omega_{p e}=\left(\frac{4 \pi n_{e} e^{2}}{m_{e}}\right)^{1 / 2}, \quad \lambda_{D}=\left(\frac{T_{e}}{4 \pi n_{e} e^{2}}\right)^{1 / 2}=\frac{v_{\mathrm{th}}}{\sqrt{2} \omega_{p e}}, \\
& v_{\mathrm{th}}=\left(\frac{2 T_{e}}{m_{e}}\right)^{1 / 2} \quad c_{S}=\left(\frac{T_{e}}{m_{i}}\right)^{1 / 2} .
\end{aligned}
$$

In the above, $\omega_{p e}$ is the plasma frequency, and $n_{e}, e$, and $m_{e}$ are the electron number density, unit electric charge, and electron mass, respectively; $\lambda_{D}$ represents the Debye length, $T_{e}$ and $T_{i}$ are the electron and ion temperatures, respectively; and $v_{\text {th }}$ and $c_{S}$ stand for electron thermal speed and ion-sound speeds, respectively, with $m_{i}$ being the proton mass.

The equations of WT theory are made of kinetic equations that govern the dynamical evolution of the electron VDF, $F_{e}(\boldsymbol{v}, t)$, and spectral wave intensities, $I_{L}^{ \pm}(\boldsymbol{k}, t), I_{S}^{ \pm}(\boldsymbol{k}, t)$, and $I_{T}^{ \pm}(\boldsymbol{k}, t)$. The governing equations for these quantities can be found in Yoon (2006), Yoon et al. (2012b), and Ziebell et al. (2015), but we particularly refer to Equations (8)-(14) of Ziebell et al. (2015). For the sake of space we do not reproduce these equations here, but we simply refer to the abovereferenced papers. The physical meanings of the various terms in Equations (8)-(14) of Ziebell et al. (2015) are listed in that study, so we will not repeat them here. Ziebell et al. (2015) numerically solved the set of Equations (8)-(14) in their paper, and by carefully turning various terms on or off, they carried out detailed diagnostics of each process. The purpose of the present analysis is not to repeat the detailed tasks already carried out by Ziebell et al. (2015), but rather to validate the efficacy of the WT simulation method by testing the numerical solutions against the PIC code simulation, which is more rigorous.

We next turn to a brief description of the PIC simulation adopted in the present paper. We have carried out a series of twodimensional relativistic and electromagnetic PIC simulations. The simulation box size is $L_{X}=L_{Y}=64.83 \mathrm{c} / \omega_{p e}=1024 v_{\text {th }} / \omega_{p e}$, where $c$ is the speed of light, $v_{\text {th }}$ is the electron thermal speed, and $\omega_{p e}$ is the electron plasma frequency. The number of grids is $N_{X}=N_{Y}=1024$, so the grid size is $\Delta x=\sqrt{2} \lambda_{D}$. The simulation time step is $\Delta t=\Delta x / 2 c$, which satisfies the Courant-FriedrichsLewy (CFL) condition, where $\Delta t$ is the time step.

The number of particles is 200 per grid per species, and we used three kinds of species: protons, background electrons, and beam electrons. The proton-to-electron mass ratio is realistic, $m_{p} / m_{e}=1836$, where $m_{p}$ is the proton mass and $m_{e}$ is the electron mass. The electron thermal speed is $v_{\text {th }}^{2} / c^{2}=4.0 \times$ $10^{-3}$. The electron temperature is seven times higher than the proton temperature, $T_{i} / T_{e}=1 / 7$, where $T_{i}$ is the ion (proton) temperature, and $T_{e}$ is the electron temperature.

The beam consists of $0.1 \%$ of the total electron content, namely, $n_{b} / n_{0}=10^{-3}$, where $n_{b}$ is the beam number density and $n_{0}$ is the electron number density. The Maxwellian beam temperature is the same as the background electron temperature, $T_{b}=T_{e}$, where $T_{b}$ is the electron beam temperature. The plasma to cyclotron frequency ratio is $\omega_{p e} / \Omega_{c e}=100$, where $\Omega_{c e}$ is electron cyclotron frequency. The boundary conditions are periodic for both the $X$ and $Y$ directions.

The above described parameters are chosen as in the paper by Ziebell et al. (2015). We have carried out three simulations where we have varied the average beam drift speed, $V_{b} / v_{\text {th }}$, from 6 , to 8 , to 10 . Again, these choices are the same ones considered by Ziebell et al. (2015).

\section{Numerical Analysis}

We solved the equations of GWT turbulence theory; that is, we carried out the WT simulation, and also carried out the PIC code simulation under the same set of initial conditions so that direct comparisons could be made, which is unprecedented. In the WT simulation we take the protons as a stationary background with the 2D VDF given by

$$
F_{i}(\boldsymbol{v})=\frac{m_{i}}{2 \pi T_{i}} \exp \left(-\frac{m_{i} v^{2}}{2 T_{i}}\right),
$$

where $T_{i}$ is the proton thermal speed.

In the PIC code, on the other hand, the protons are free to dynamically evolve, as there are no reasons to fix the protons as a neutralizing background. The initial electron VDF is the same as that adopted in the analysis by Ziebell et al. (2015), namely, the electrons are composed of a backward drifting background Maxwellian component plus a tenuous forward drifting Maxwellian distribution of electron beams, which, in two dimensions, is given by

$$
\begin{gathered}
F_{e}(\boldsymbol{v}, 0)=\left(1-\frac{n_{b}}{n_{0}}\right) \frac{m_{i}}{2 \pi T_{e}} \exp \left(-\frac{m_{e} v_{\perp}^{2}}{2 T_{e}}-\frac{m_{e}\left(v_{\|}+V_{0}\right)^{2}}{2 T_{e}}\right) \\
+\frac{n_{b}}{n_{0}} \frac{m_{e}}{2 \pi T_{b}} \exp \left(-\frac{m_{e} v_{\perp}^{2}}{2 T_{b}}-\frac{m_{e}\left(v_{\|}-V_{b}\right)^{2}}{2 T_{b}}\right) .
\end{gathered}
$$

We can define the thermal speeds associated with each component in their respective drifting frame as $v_{\text {th }}=$ $\left(2 T_{e} / m_{e}\right)^{1 / 2}$ and $v_{t b}=\left(2 T_{b} / m_{e}\right)^{1 / 2}$. Here, and $V_{0}$ and $V_{b}$ are the drift velocities of the background and the forward beams, respectively, where the background drift velocity $V_{0}$ is assumed in order to preserve the zero current condition in the proton frame,

$$
V_{0}=\frac{n_{b} V_{b}}{n_{0}-n_{b}}
$$

The PIC code simulation also initialized the electron configuration in the same manner as in the analytical initial VDF. The initial spectral forms for the $L, S$, and $T$ mode intensities in the WT simulation are chosen as in Ziebell et al. (2015),

$$
\begin{aligned}
I_{L}^{\sigma}(\boldsymbol{k}, 0)= & \frac{T_{e}}{4 \pi^{2}} \frac{1}{1+3 k^{2} \lambda_{D e}^{2}}, \\
I_{S}^{\sigma}(\boldsymbol{k}, 0)= & \frac{T_{e}}{4 \pi^{2}} k^{2} \lambda_{D e}^{2}\left(\frac{1+k^{2} \lambda_{D e}^{2}}{1+3 k^{2} \lambda_{D e}^{2}}\right)^{1 / 2} \\
& \times \frac{\int d \boldsymbol{v} \delta\left(\sigma \omega_{\boldsymbol{k}}^{S}-\boldsymbol{k} \cdot \boldsymbol{v}\right)\left(F_{e}+F_{i}\right)}{\int d \boldsymbol{v} \delta\left(\sigma \omega_{\boldsymbol{k}}^{S}-\boldsymbol{k} \cdot \boldsymbol{v}\right)\left[F_{e}+\left(T_{e} / T_{i}\right) F_{i}\right]} \\
I_{T}^{\sigma}(\boldsymbol{k}, 0)= & \frac{T_{e}}{2 \pi^{2}} \frac{1}{1+c^{2} k^{2} / \omega_{p e}^{2}} .
\end{aligned}
$$

Of course, in the PIC code simulation, the initial noise is present in the system so that one does not need to specify the initial spectral form for the modes. 
The initial input parameters are taken to be the same as that considered by Ziebell et al. (2015). These are

$$
\begin{aligned}
\frac{1}{n_{e} \lambda_{D}^{3}} & =5 \times 10^{-3}, \quad T_{e}=T_{b}, \\
\frac{T_{i}}{T_{e}} & =\frac{1}{7}, \quad \frac{v_{\mathrm{th}}^{2}}{c^{2}}=\frac{2 T_{e}}{m_{e} c^{2}}=4.0 \times 10^{-3}, \\
\frac{n_{b}}{n_{0}} & =10^{-3} .
\end{aligned}
$$

Among the above input parameters, the plasma parameter, $1 /\left(n \lambda_{D}^{3}\right)$, is strictly applicable only for the WT simulation. As we already explained in the previous section, the PIC code simulation was designed with the same specification as (9), except for the plasma parameter. In the PIC code, the number of particles per cell is loosely connected to the plasma parameter, as recently demonstrated by López \& Yoon (2018), but there is no precise way to strictly associate the value of the plasma parameter to the PIC code design parameters such as the dimensions, the number of particles per cell, etc.

We adopt the normalized wave vector, velocity, and time,

$$
\frac{\boldsymbol{k} v_{\mathrm{th}}}{\omega_{p e}}, \quad \frac{\boldsymbol{v}}{v_{\mathrm{th}}}, \quad \omega_{p e} t,
$$

in plotting the numerical results. Of course, the proton-toelectron mass ratio is realistic, $m_{i} / m_{e}=1836$. Ziebell et al. (2015) considered three cases of normalized forward beam speed, $V_{b} / v_{\text {th }}=6,8$, and 10 . The PIC code simulations are carried out with the same conditions so that direct comparisons can be made.

In the following, we present quantitative comparisons of the results obtained by solving the equations of WT theory, that is, the WT simulation, and the PIC code simulation. In the WT analysis, where we revisited the earlier approach, we considered a wider velocity space compared to that of Ziebell et al. (2015), with the objective of minimizing the effect of boundary conditions. We present the results in the same format as in the paper by Ziebell et al. (2015), except that we choose to present only the snapshots of electron VDF, Langmuir wave spectrum, and transverse wave spectrum, or equivalently, the radiation pattern, as these quantities are dynamically important. The ion-acoustic mode is excited in both PIC code simulations and WT theory, but as their wave intensities are generally low, we choose not to plot the results. In the PIC code simulation, in particular, fluctuations in the ion-acoustic wave frequency range are too weak to distinguish them from numerical noise. It should also be noted that the theoretical ion-acoustic mode turbulence intensity discussed in the paper by Ziebell et al. (2015) is also very low. We thus focus on those quantities that may be meaningfully compared, namely electron VDF, highfrequency longitudinal electric field intensity in the Langmuir wave frequency range, a perturbed magnetic field spectrum constructed from a PIC simulation, and the corresponding theoretical electron velocity distribution, Langmuir wave intensity, and transverse radiation intensity computed on the basis of WT theory.

\subsection{Case 1: $V_{b} / v_{t h}=6$}

In Figure 1 we show the electron VDF computed on the basis of the WT simulation (top four panels) and the electron velocity phase space distribution constructed from the PIC code simulation (bottom four panels), versus two-dimensional normalized perpendicular and parallel speeds, $v_{\perp} / v_{\text {th }}$ and $v_{\|} / v_{\text {th }}$, for the first case of $V_{b} / v_{\text {th }}=6$ (case 1). Snapshots of the electron VDF at four different times corresponding to $\omega_{p e} t=500,1000,1500$, and 2000 are shown.

On the basis of the direct comparisons between the theory, or WT simulation, and PIC simulation, it can be seen that there exists an overall quantitative, and even qualitative agreement between the two methods. Specifically, for $\omega_{p e} t=500$, both methods produce similar results in that the beam has undergone a partial plateau formation, but a significant positive gradient, $\partial F_{e} / \partial v_{\|}>0$ still exists along the parallel velocity. As time progresses the positive slope is gradually reduced, until at $\omega_{p e} t=2000$, the plateau formation is almost complete in the case of WT simulation. By contrast, for the PIC code case, a small but finite positive gradient still persists at $\omega_{p e} t=2000$. Despite this rather insignificant difference, the two methods agree quite well.

The Langmuir turbulence spectrum is shown in Figure 2. In the top four panels, the Langmuir spectral intensity $I_{L}(\boldsymbol{k})$ is plotted versus $k_{\perp} v_{\text {th }} / \omega_{p e}$ and $k_{\|} v_{\text {th }} / \omega_{p e}$. In computing for the forward and backward modes, we restricted ourselves to only positive $k_{\|}>0$ space, but when we plot the final results, we combined both signs of $\sigma= \pm 1$ in one panel by showing $I_{L}^{+1}(\boldsymbol{k})$ in $k_{\|}>0$ space, while showing $I_{L}^{-1}(\boldsymbol{k})$ in the negative $k_{\|}$space. Thus, the portion of $\boldsymbol{k}$ space corresponding to $k_{\|}>0$ should be interpreted as $I_{L}^{+1}(\boldsymbol{k})$, while the other half-space with $k_{\|}<0$ is the backward $L$ mode, $I_{L}^{-1}(\boldsymbol{k})$. For the bottom four panels, which correspond to the simulated electric field spectral intensity, it is not as easy to single out the contribution from the eigenmode, which is what the $L$ mode is. Instead, we have filtered out the low- and highfrequency fluctuations, and focused only on the spectrum that roughly encompasses the plasma frequency. Note, however, that the spectrum in the vicinity of plasma frequency not only contains the Langmuir mode intensity, which is the linear eigenmode of the plasma, but also nonlinear eigenmode; see Rhee et al. (2009b). The simulated electric field spectrum does not distinguish between the two. The total electric field may also contain the radiation mode, but in order to eliminate the transverse component as much as possible, we have selected only the electric field component parallel to the beam propagation direction before implementing the fast Fourier transformation of the simulated electric field. These subtle differences notwithstanding, a comparison between the theoretical intensity and simulated spectrum shows a rather striking resemblance between them both.

Specifically, for early time, $\omega_{p e} t=500$, the enhanced forward-propagating component (the primary $L$ ) can be seen to be excited in both WT and PIC simulations, which is the result of an initial gentle bump-on-tail instability. At this relatively early time the weak backward propagating $L$ mode is also evident. For $\omega_{p e} t=1000$ and beyond, the backscattering of the primary $L$ mode into the backscattered $L$ mode, via a combined three-wave decay process and nonlinear scattering of ions, becomes increasingly more visible (Ziebell et al. 2001, 2008a, 2012, 2014b, 2015). In an overall sense, the characteristic timescale of wave evolution and the spectral feature at each stage of time evolution, in particular, the formation of semi-ark shape spectra in both the WT and PIC 

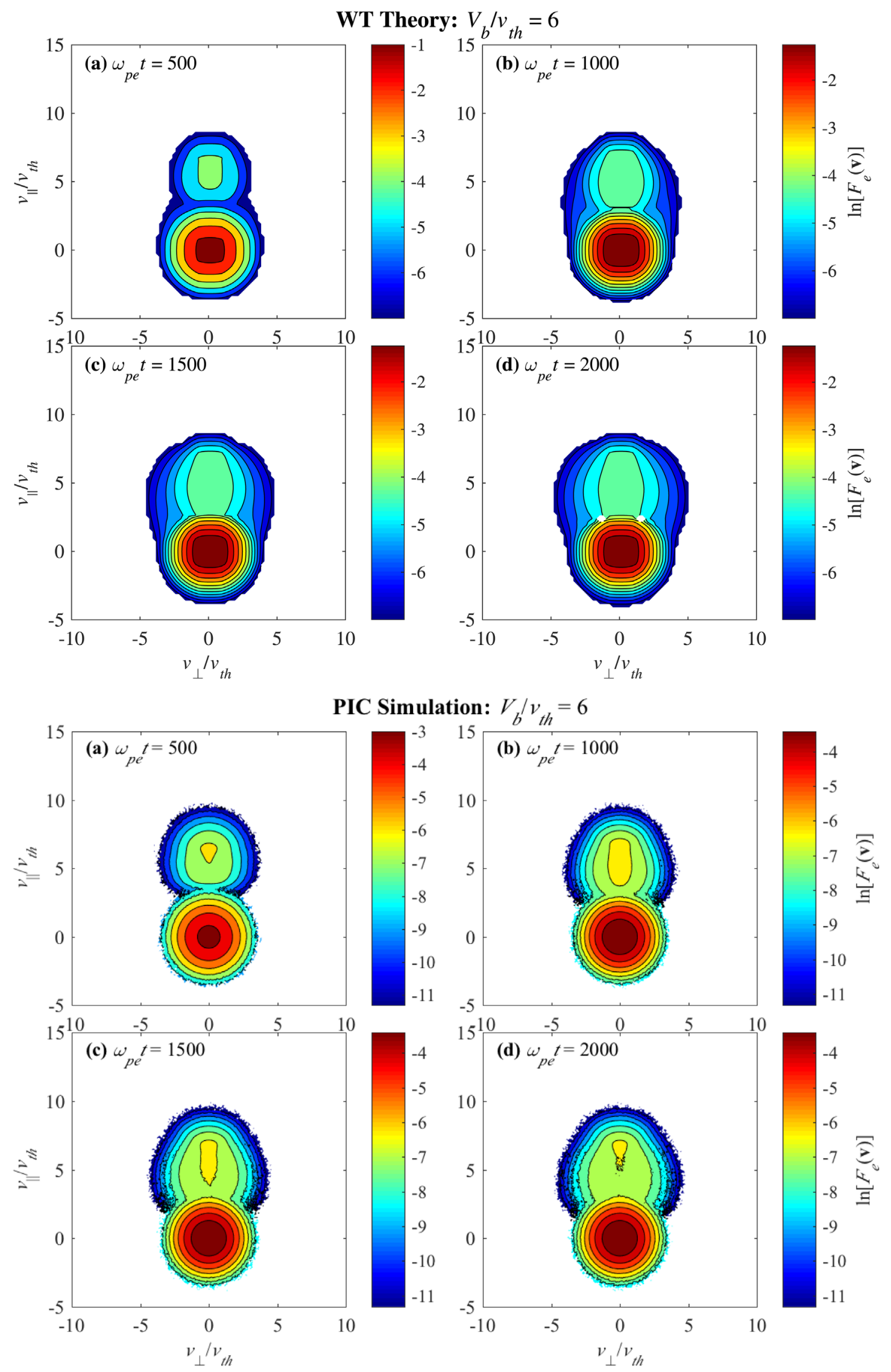

Figure 1. Case $1\left(V_{b} / v_{\text {th }}=6\right)$ : time evolution of the electron velocity distribution function (VDF) $F_{e}(v)$ vs. $v_{\perp} / v_{\text {th }}$ and $v_{\|} / v_{\text {th }}$, for four different time steps corresponding to $\omega_{p e} t=500,1000,1500$, and 2000. The top four panels correspond to the WT simulation, while the bottom four panels show results from the PIC code simulation. 


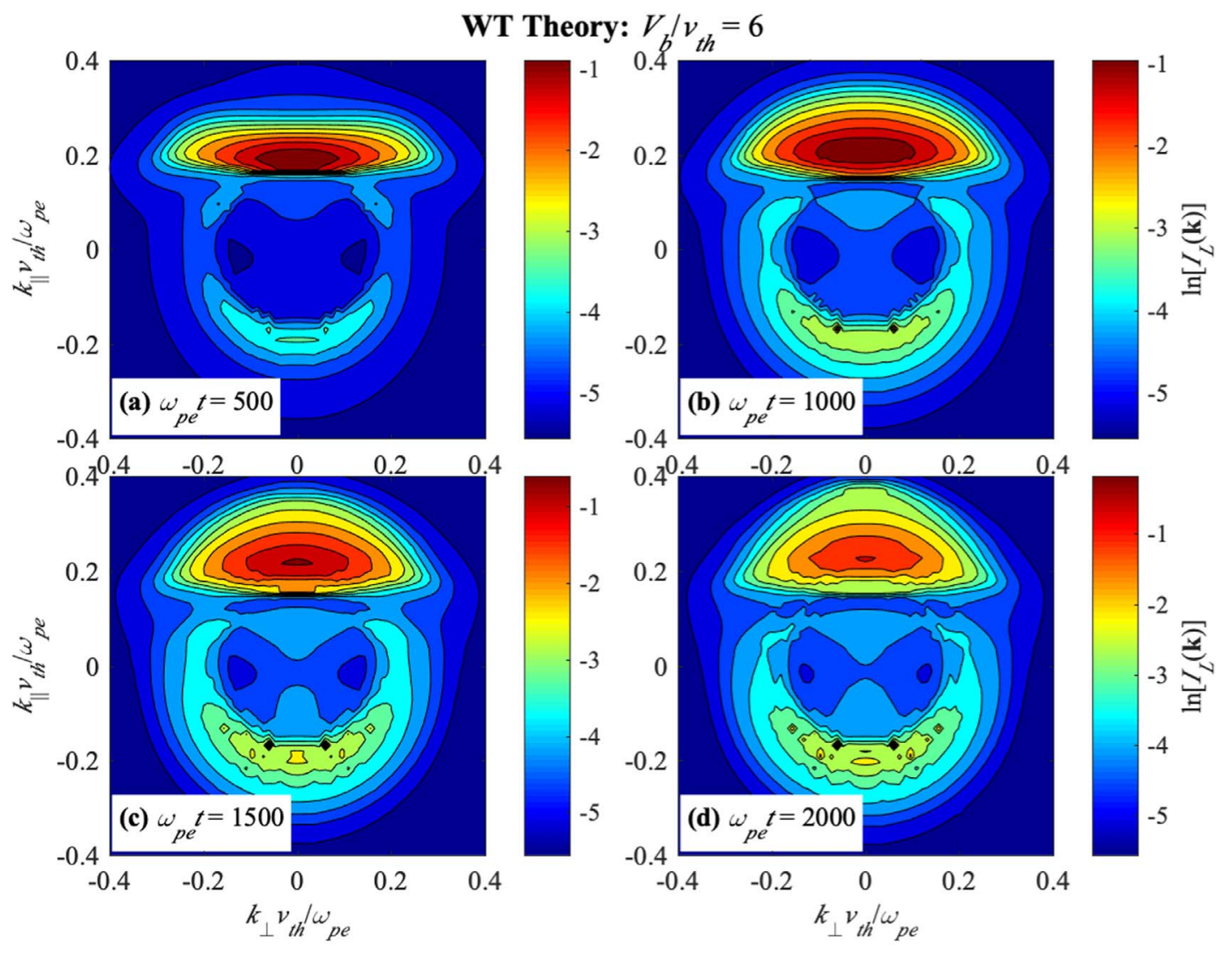

PIC Simulation: $V_{b} / v_{t h}=6$
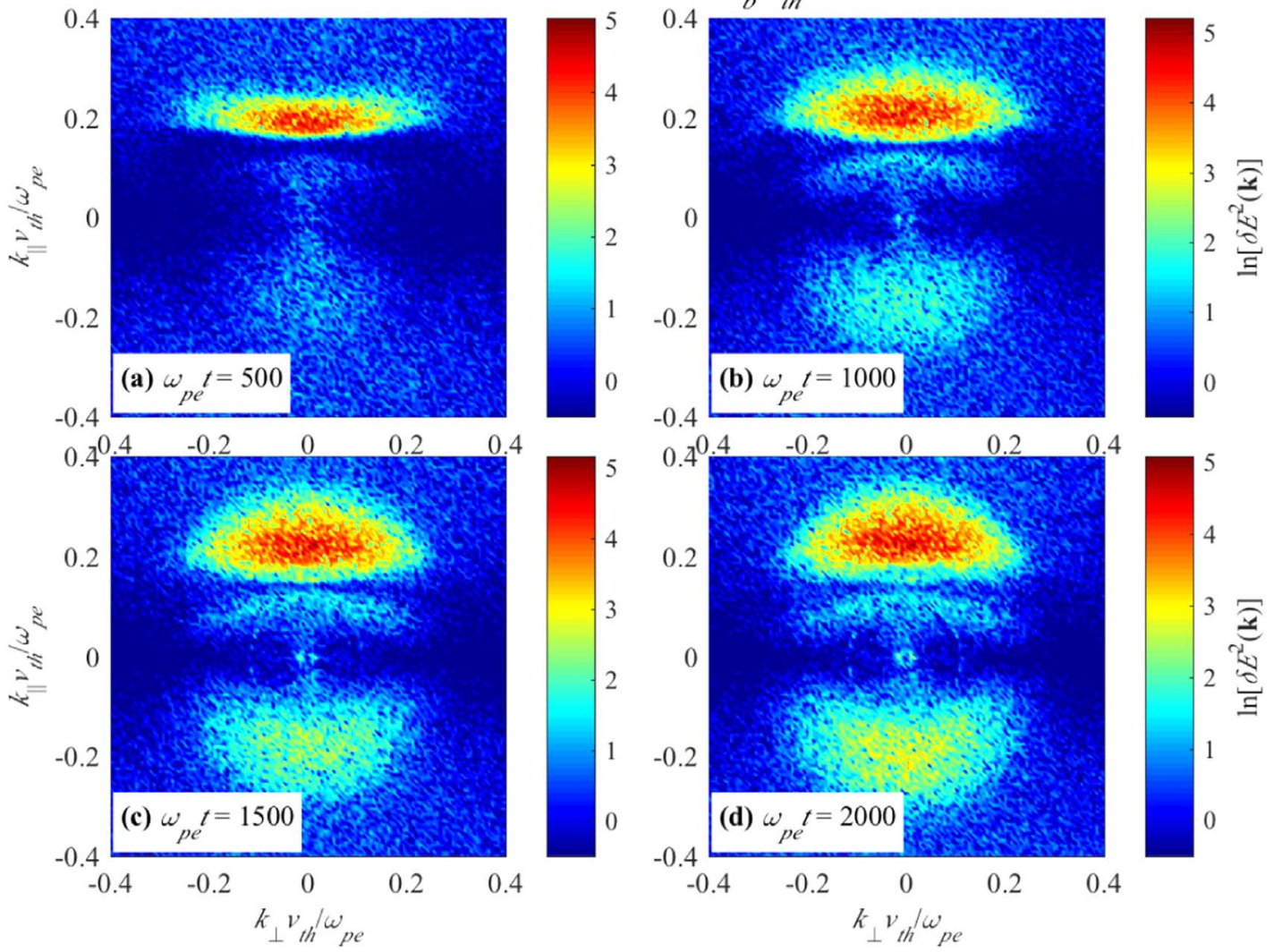

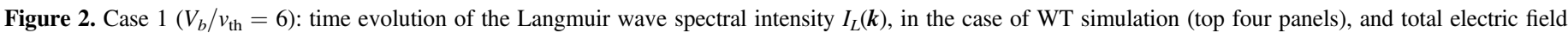
intensity $\delta E^{2}(\boldsymbol{k})$, in the case of PIC code simulation (bottom four panels), vs. $k_{x} v_{\text {th }} / \omega_{p e}$ and $k_{z} v_{\text {th }} / \omega_{p e}$, for $\omega_{p e} t=500,1000$, 1500 , and 2000 . 
code simulations, are quite consistent. One minor difference is that, whereas in the WT calculation, growth of the near $k \sim 0$ mode, that is, the Langmuir condensation, is not apparent, the PIC code quite readily indicates that the condensation of Langmuir wave energy to long wavelength mode takes place early on. It has been shown that the effect of spontaneous electron scattering contributes to scattering to the region of low wave numbers, excluding the region $k \simeq 0$ (Ziebell et al. 2012). The present calculation does include such an effect, but apparently, it is not sufficient to account for the formation of Langmuir condensation effect.

Figure 3 plots the transverse EM radiation ( $T$ mode) spectrum computed on the basis of the WT theoretical method (top four panels), and the magnetic field spectrum constructed from the PIC simulation (bottom four panels). According to the WT calculation, for early time $\omega_{p e} t=500$, a finite level of fundamental emission is first generated. Then, at $\omega_{p e} t=1000$ and beyond, a second-harmonic mode gradually appears until at $\omega_{p e} t=2000$, the fundamental/harmonic bi-modal radiation pattern is established. In contrast, in the present case of weak beam speed, $V_{b} / v_{\text {th }}=6$, the magnetic field spectrum is dominated by the noise almost throughout the entire simulated time domain. Only at the very end, $\omega_{p e} t=2000$, does a weak signature of fundamental/harmonic pair emission structure become barely discernible. This seems to indicate the need for a higher number of particles per cell in order to reduce the numerical noise.

\subsection{Case 2: $V_{b} / v_{t h}=8$}

Ziebell et al. (2015) also considered the second case corresponding to $V_{b} / v_{\text {th }}=8$ (case 2 ), which we consider next. Figure 4 displays the electron VDF in the same format as Figure 1. As with case 1, the time evolution of electron VDF is qualitatively similar for both WT and PIC simulations, especially for relatively early time, $\omega_{p e} t=500$. Note, however, that as the system evolves in time, while the qualitative agreement is still maintained, upon close inspection, some quantitative discrepancies become noticeable. Most appreciable is the fact that while the two-dimensional structure associated with the theoretically computed electron VDF is defined by elliptical outer contours for the beam population, the PIC simulated electron VDF exhibits a distinct broadening of the beam along $v_{\perp}$, while the highest parallel velocity portion of the beam population does not suffer from such broadening. Another feature that is noteworthy is that for $\omega_{p e} t=2000$, the theoretical VDF still shows a mild positive parallel derivative associated with the beam, whereas for the simulated VDF such a feature is almost completely gone. In spite of these, it is quite fair to say that the WT simulation features a good overall comparison against the PIC simulation.

The Langmuir turbulence spectrum and the simulated longitudinal electric field spectrum are plotted in Figure 5. Again, despite the subtle differences in the two quantities plotted, as already explained (namely, the theoretical quantity is the Langmuir wave intensity while the simulated quantity is longitudinal electric field fluctuation centered around the plasma oscillation frequency, which may contain both the Langmuir mode as well as the nonlinear eigenmode), the overall agreement is rather remarkable. Upon direct comparison, it is seen that the contours for both the theoretical spectrum and the simulated intensity evolve into a more or less ring-like morphology in 2D $\boldsymbol{k}$ space. For the present case of higher beam speed, more free energy is available for the excitation of Langmuir instability. Consequently, the entire instability and ensuing nonlinear processes develop much more rapidly. This results in many back-and-forth decay and scattering processes, which leads to the multiply-peaked structures in the wave spectrum along $k_{\|}$. This is particularly apparent in the WT simulation. In the PIC code simulation, on the other hand, owing to the inherent noise, the clear delineation of multiplepeak structure along $k_{\|}$is not so evident. However, upon close examination, especially for $\omega_{p e} t=2000$, the forwardpropagating longitudinal mode does indeed show faint evidence of the structure along the parallel wave number. As with the first case of $V_{b} / v_{\text {th }}=6$, the PIC code simulation exhibits a rather robust Langmuir condensation phenomenon, while the WT simulation shows very weak or no evidence of Langmuir condensation.

Moving on to the transverse EM radiation ( $T$ mode) for the WT simulation, and the transverse magnetic field fluctuation spectrum for the PIC simulation, Figure 6 plots these spectra. In the present case of $V_{b} / v_{\text {th }}=8$, the fundamental plus weak harmonic emission already takes place at $\omega_{p e} t=500$, in the case of WT calculation. The pair emission pattern gradually and monotonically increases in intensity until the end of the computation, namely, $\omega_{p e} t=2000$. In contrast, as with the previous case, the PIC simulation shows no identifiable radiation emission pattern for relatively early times, $\omega_{p e} t=500$ and 1000. Even at $\omega_{p e} t=1500$, the pair emission pattern becomes barely visible with great difficulty. However, at the end of the simulation period, $\omega_{p e} t=2000$, the radiation emission pattern now becomes quite discernible over the background noise. Again, the present PIC code simulation study implies difficulty in faithfully simulating the plasma emission radiation, and calls for a higher number of particles per cell and thus a quieter simulation, which is needless to say more computationally demanding, and is beyond the scope of the present work. In this regard, the WT simulation is advantageous, because such an approach is free from the noise issue.

$$
\text { 3.3. Case 3: } V_{b} / v_{t h}=10
$$

The third case study is for $V_{b} / v_{\text {th }}=10$ (case 3 ), which was considered by Ziebell et al. (2015). In their paper, the authors speculated that the applicability of WT theory, and more specifically, the use of a weak-growth rate formula inherent in the standard WT formalism might be suspect, but they did not have any standard to verify such a suspicion. For the present relatively high beam speed, their numerical solution for the electron VDF featured a particularly undesirable aspect of the velocity plateau spreading widely until it reached the boundary, where the boundary effect began to influence the solution. In the present study, we have a benchmark tool to check the validity of the WT scheme. In Figure 7 (top four panels) we plot the electron VDF in the same format as before. We have carefully regenerated the solution with a much wider boundary in order to avoid the boundary effects. The case of $\omega_{p e} t=2000$, which appears to show that the beam has spread to the upper boundary of the figure, is actually not a problem because the actual velocity boundary is much wider than what is shown in the figure. In the bottom panels of Figure 7, we display the results of a PIC code simulation. As the comparison readily shows, the WT simulation enjoys at least a quantitative agreement, but only in the relative early time periods 


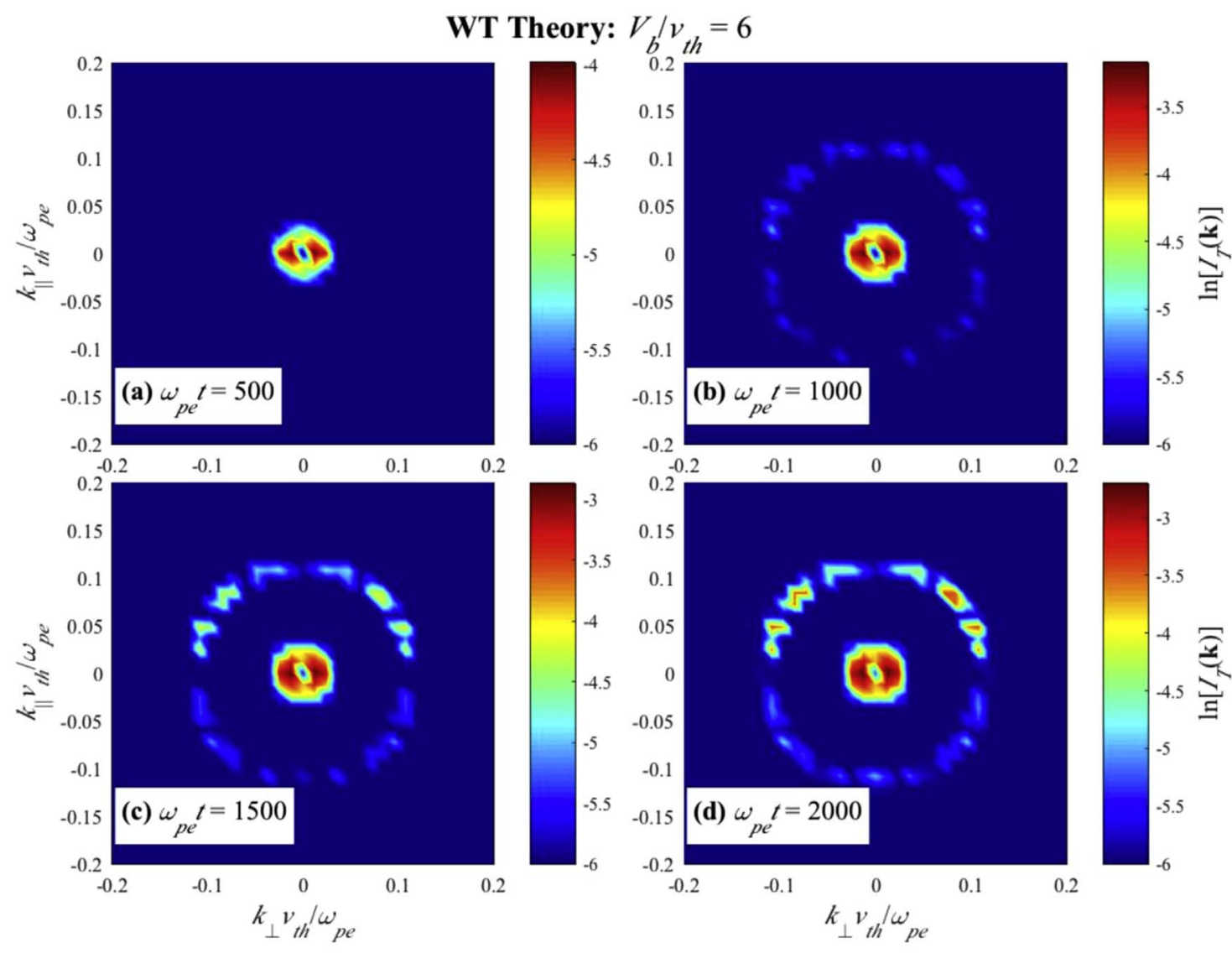

PIC Simulation: $V_{b} / v_{t h}=6$
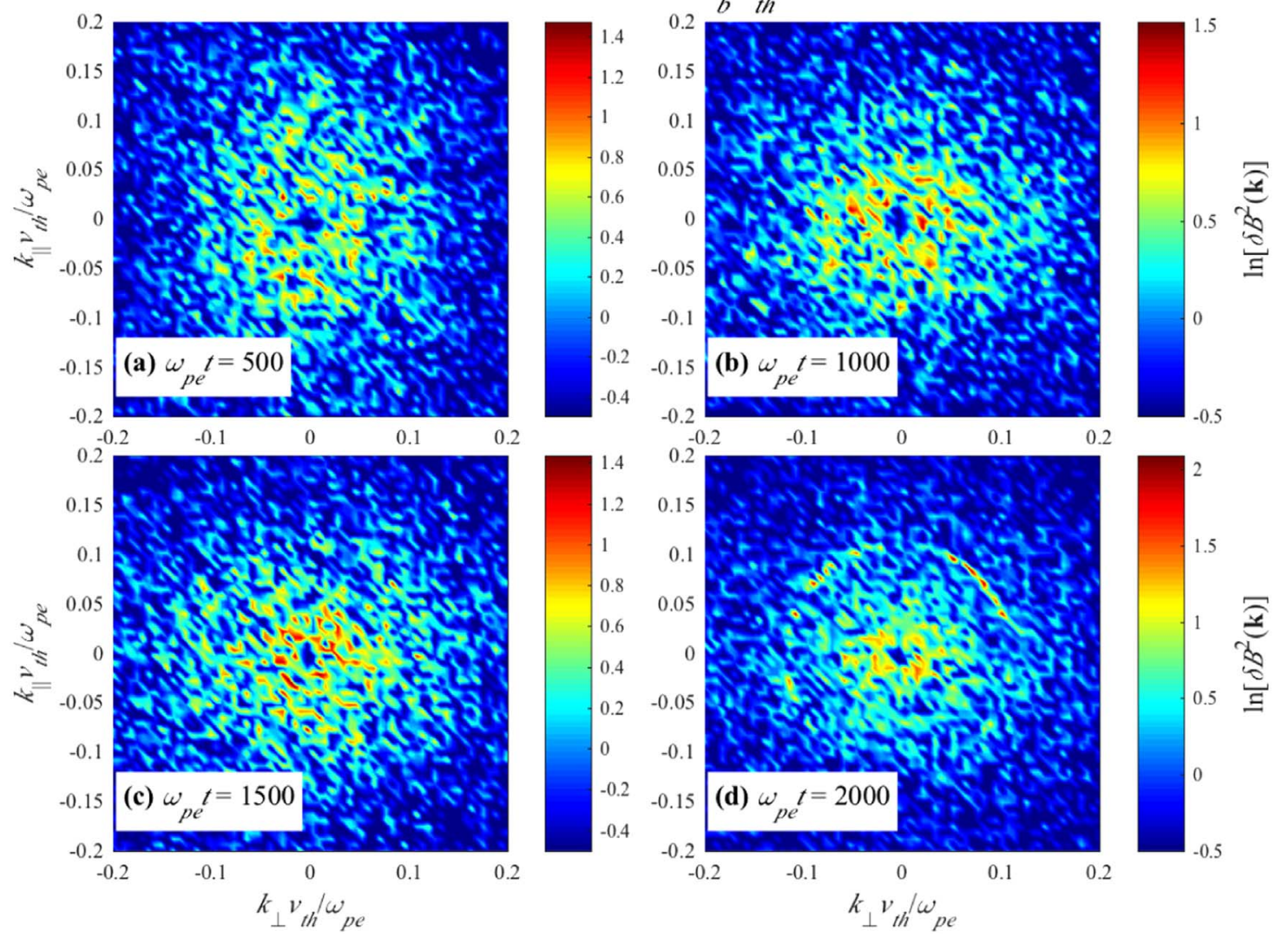

Figure 3. Case $1\left(V_{b} / v_{\text {th }}=6\right)$ : time evolution of the radiation spectral intensity $I_{T}(\boldsymbol{k})$, in the case of WT simulation (top four panels), and total magnetic field intensity $\delta B^{2}(\boldsymbol{k})$, in the case of PIC code simulation (bottom four panels), vs. $k_{x} v_{\mathrm{th}} / \omega_{p e}$ and $k_{z} v_{\mathrm{th}} / \omega_{p e}$, for $\omega_{p e} t=500,1000,1500$, and 2000. 

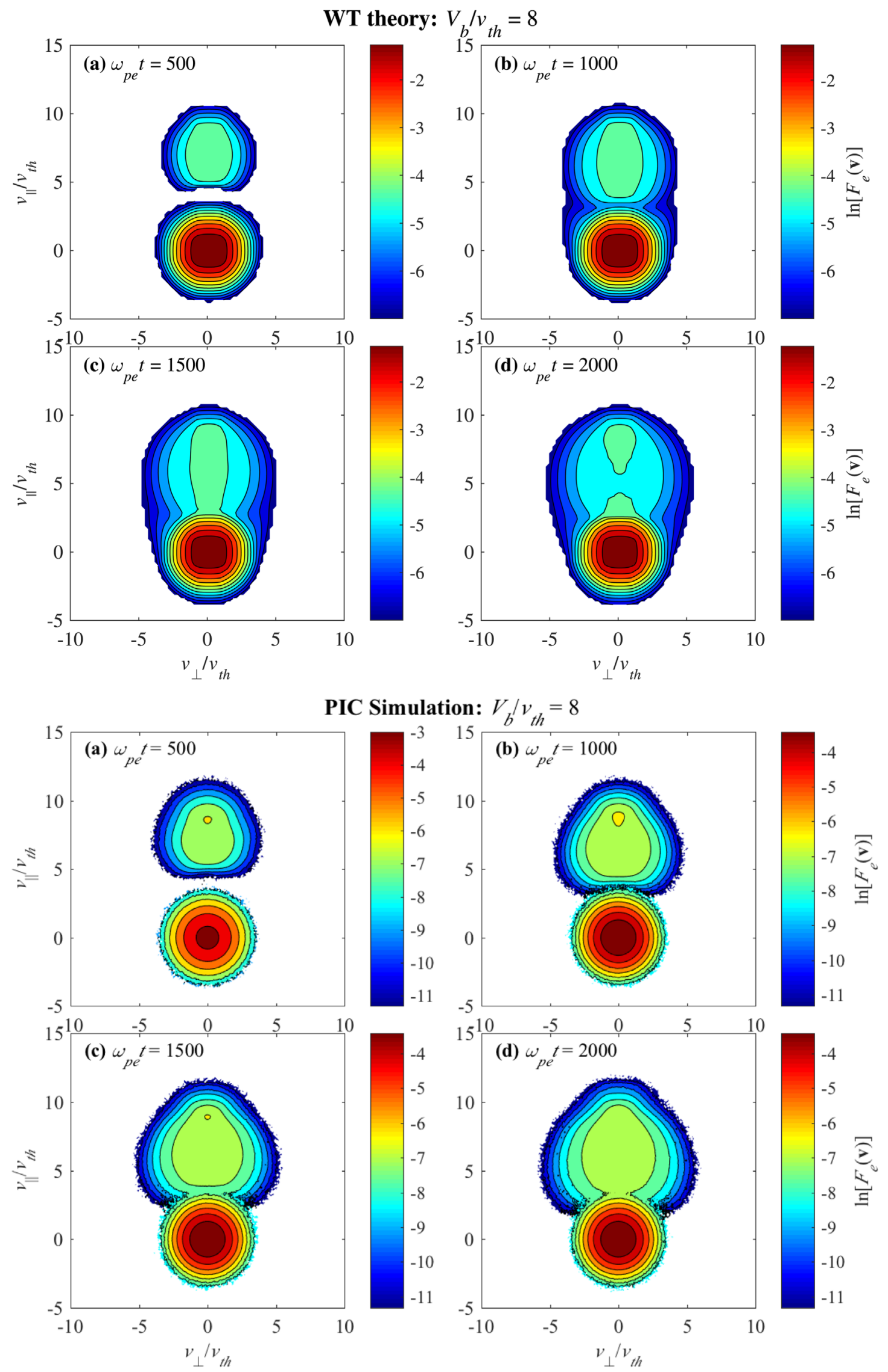

Figure 4. Case $2\left(V_{b} / v_{\mathrm{th}}=8\right)$ : electron velocity distribution function (VDF) $F_{e}(v)$ vs. $v_{\perp} / v_{\mathrm{th}}$ and $v_{\|} / v_{\mathrm{th}}$, for four different time steps corresponding to $\omega_{p e} t=500$, 1000,1500 , and 2000. The top four panels correspond to the WT simulation, while the bottom four panels show results from the PIC code simulation. 


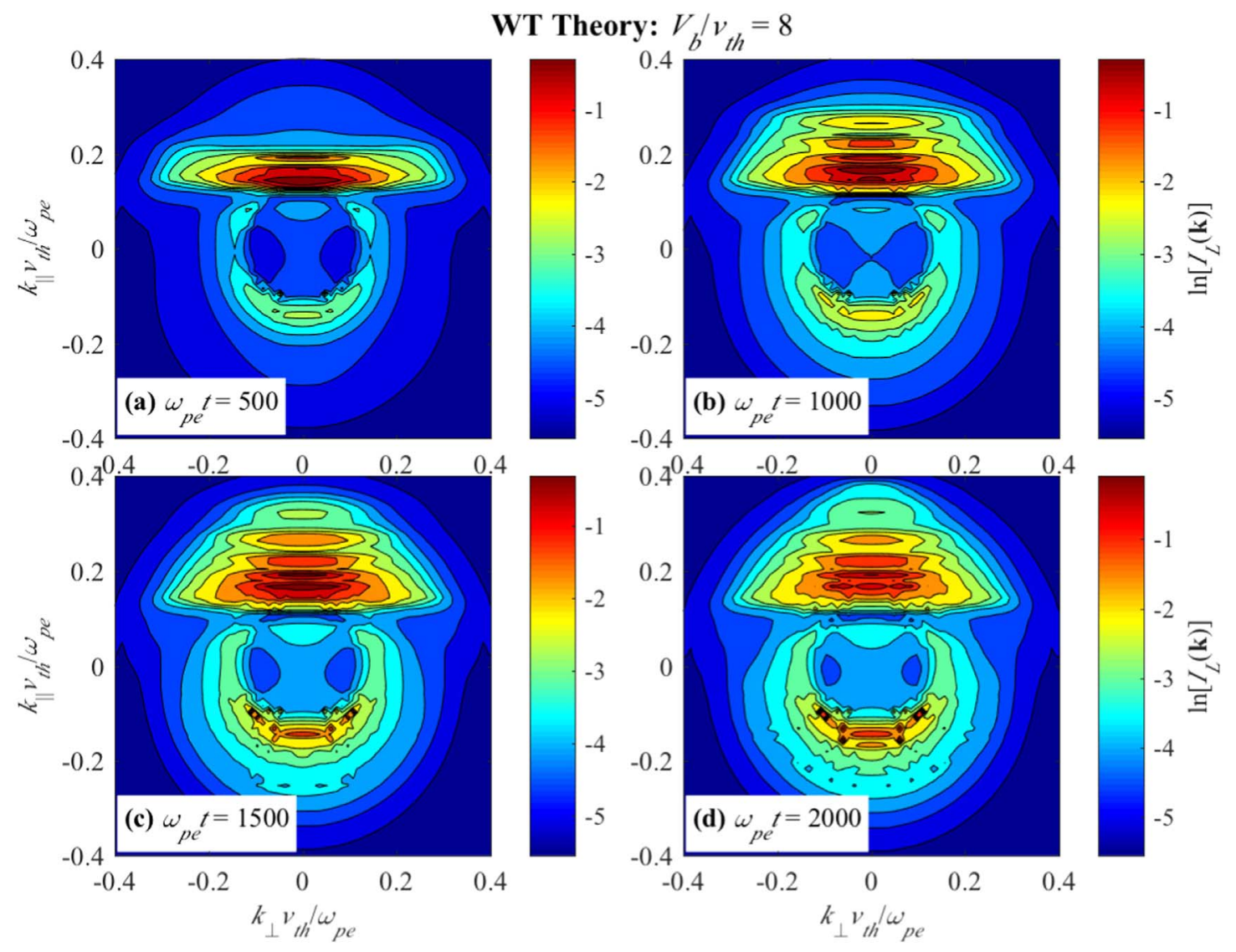

PIC Simulation: $V_{b} / v_{t h}=8$
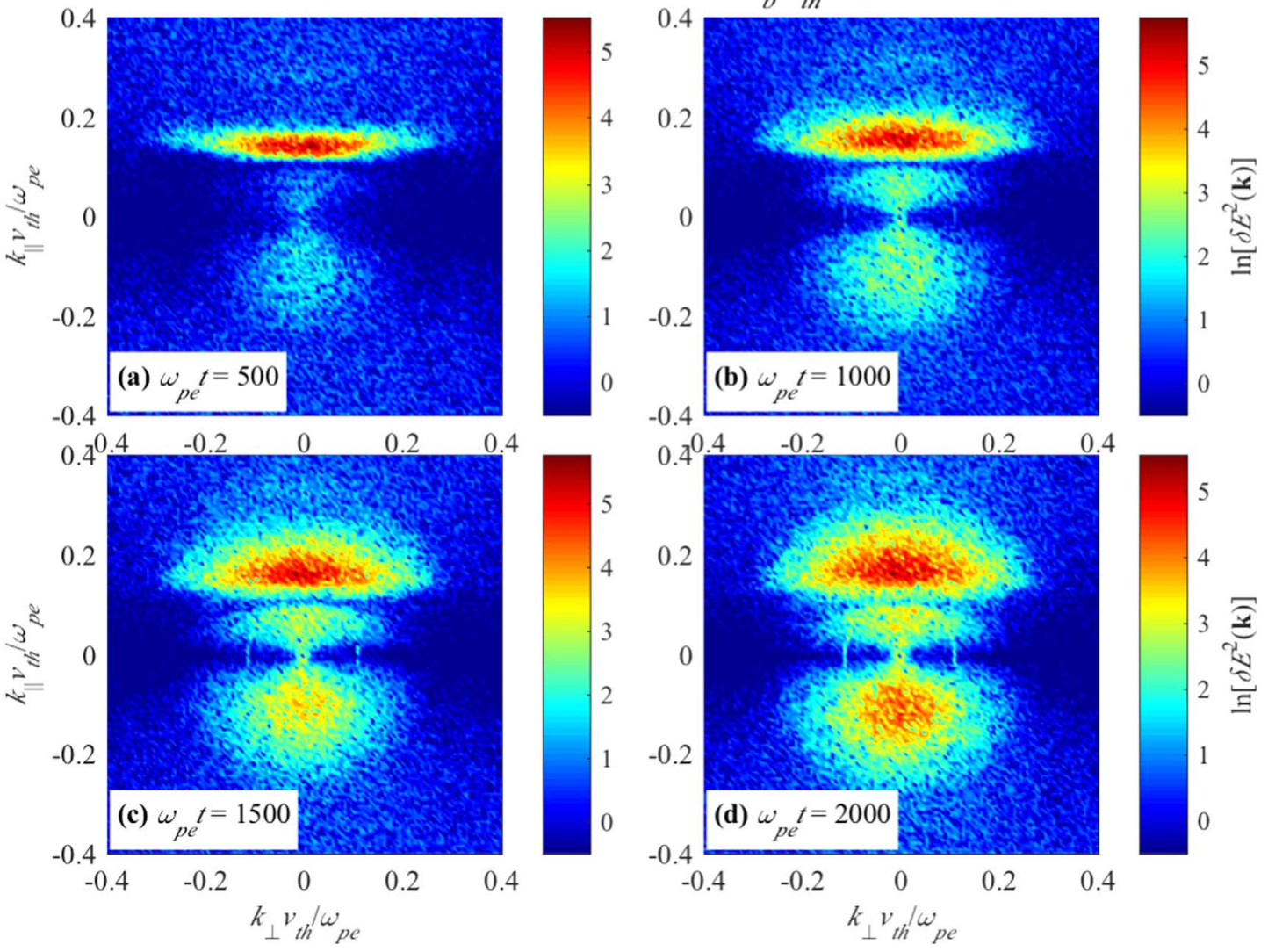

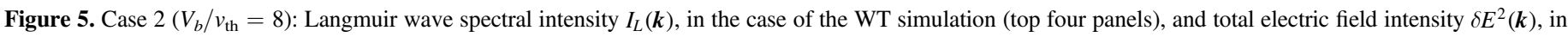
the case of the PIC code simulation (bottom four panels), vs. $k_{x} v_{\text {th }} / \omega_{p e}$ and $k_{z} v_{\text {th }} / \omega_{p e}$, for $\omega_{p e} t=500,1000,1500$, and 2000. 


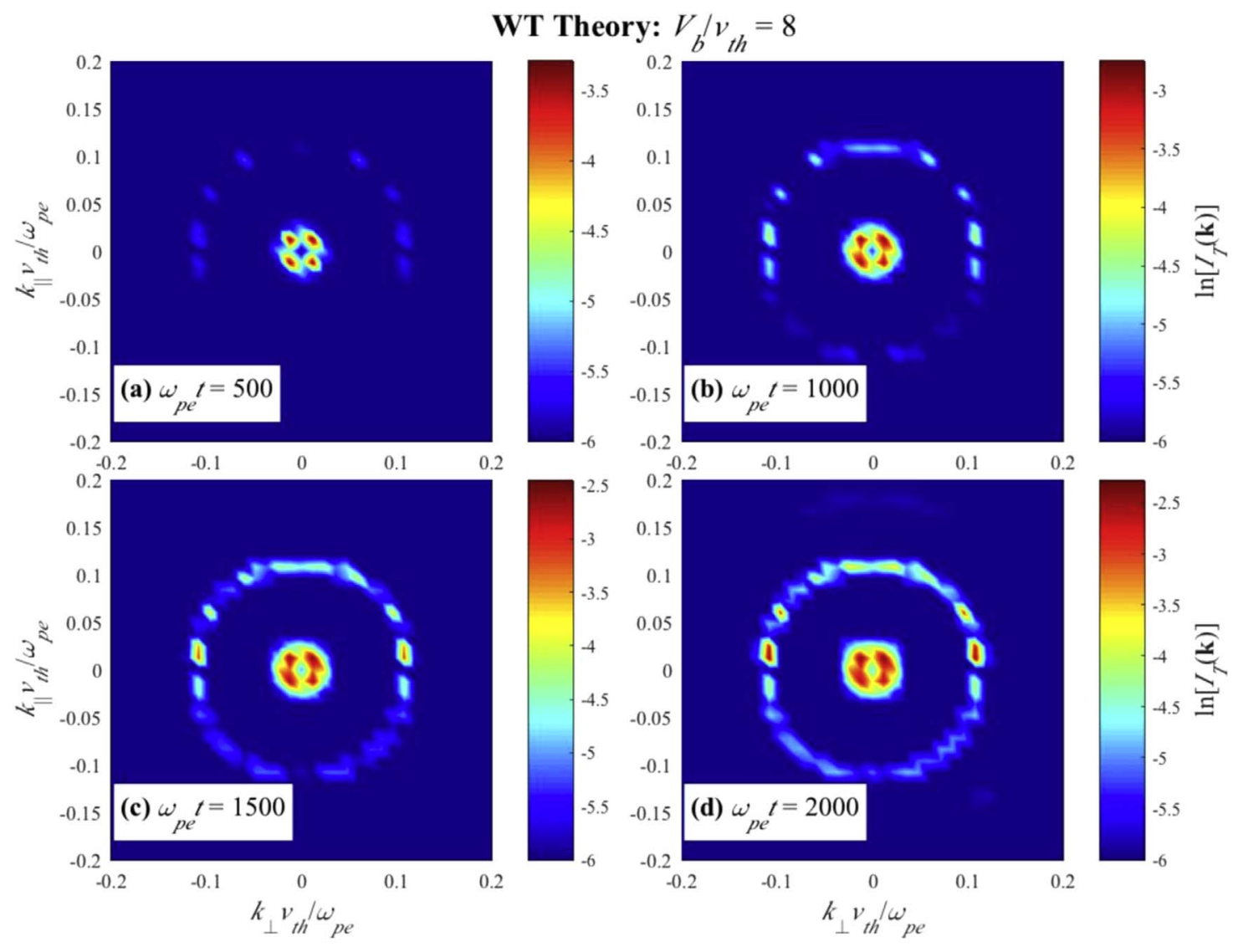

PIC Simulation: $V_{b} / v_{t h}=8$
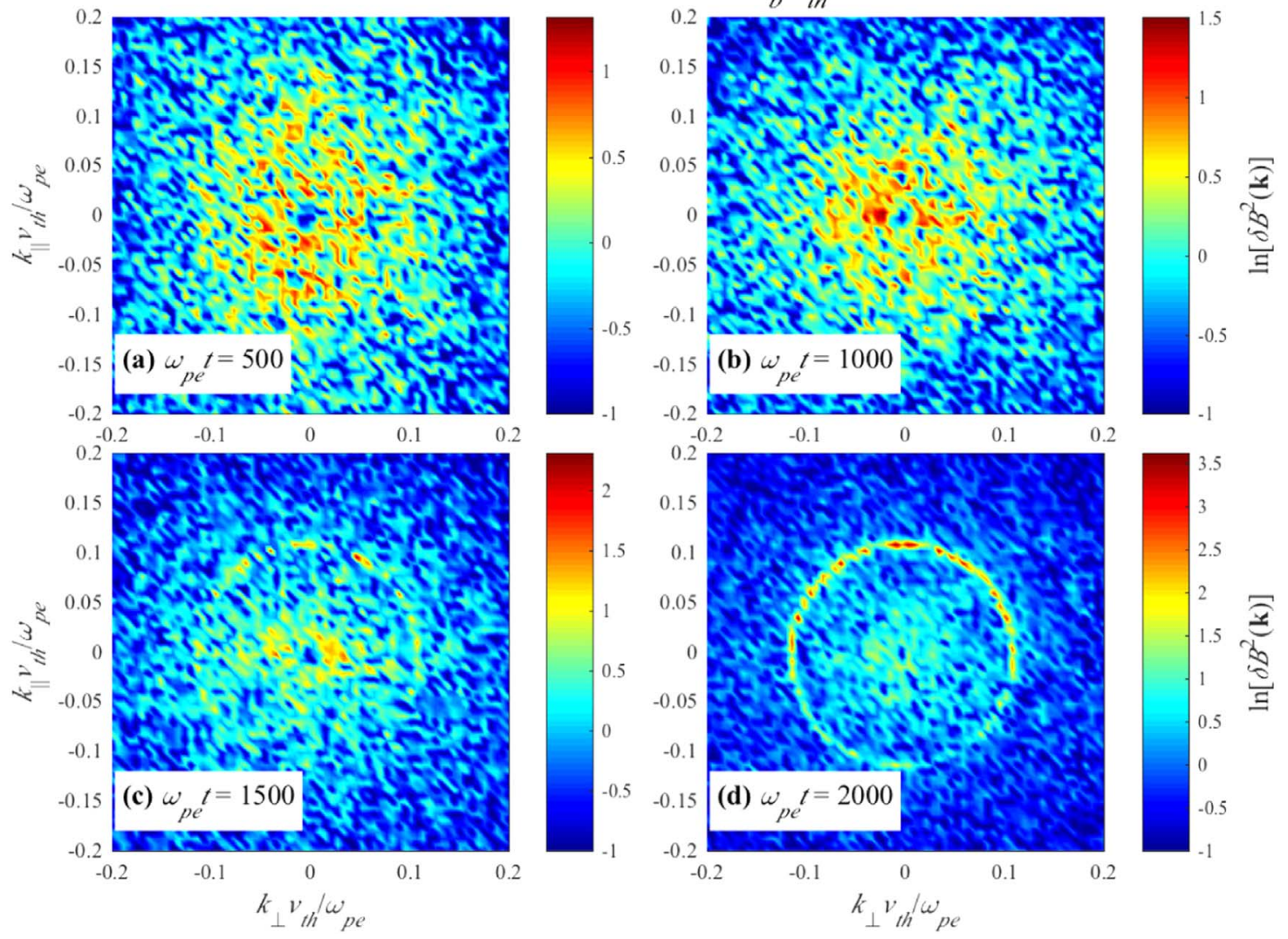

Figure 6. Case $2\left(V_{b} / v_{\text {th }}=8\right)$ : radiation spectral intensity $I_{T}(\boldsymbol{k})$, in the case of the WT simulation (top four panels), and total magnetic field intensity $\delta B^{2}(\boldsymbol{k})$, in the case of the PIC code simulation (bottom four panels), vs. $k_{x} v_{\text {th }} / \omega_{p e}$ and $k_{z} v_{\text {th }} / \omega_{p e}$, for $\omega_{p e} t=500,1000,1500$, and 2000. 

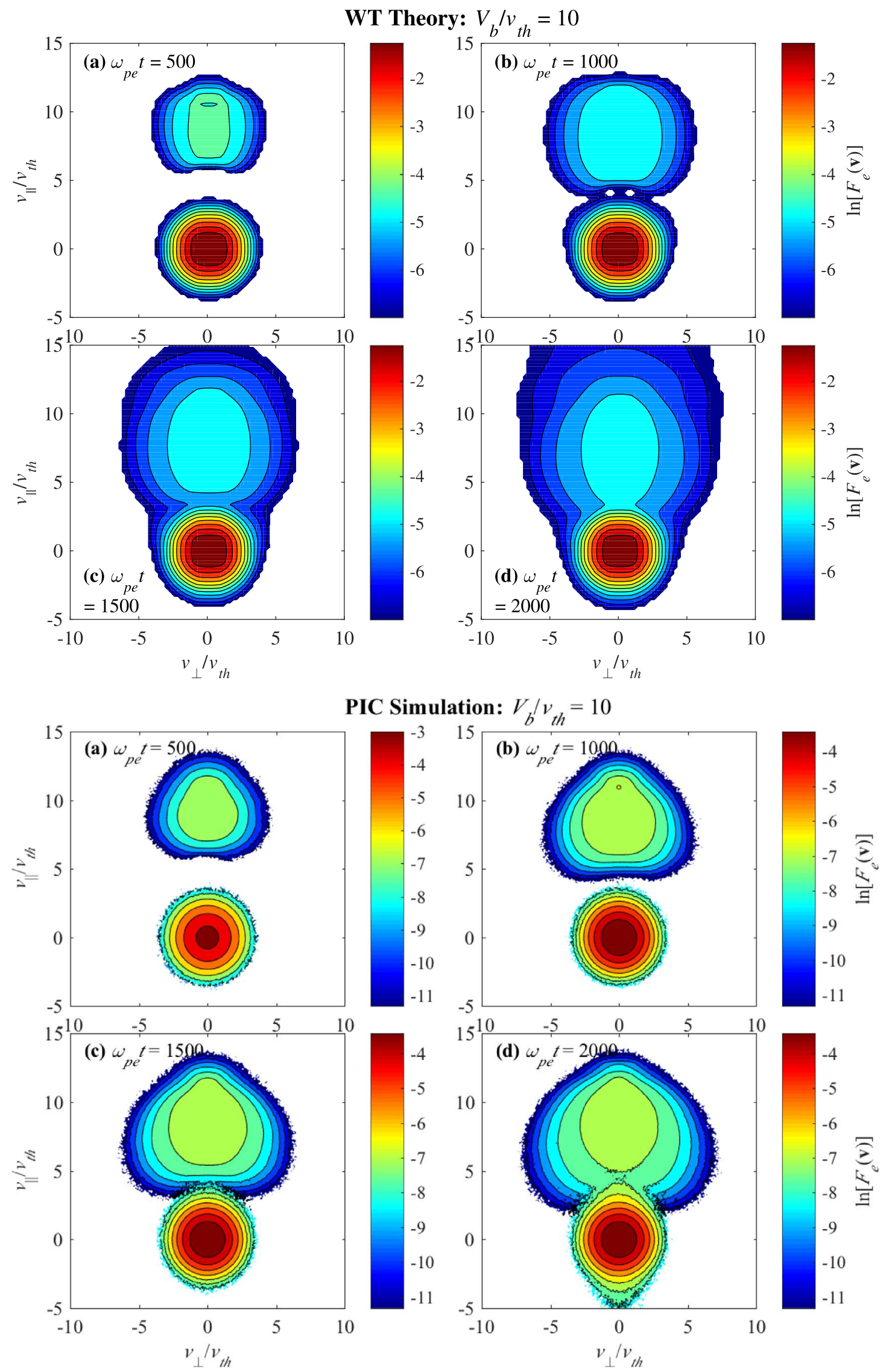

Figure 7. Case $3\left(V_{b} / v_{\mathrm{th}}=10\right)$ : electron velocity distribution function (VDF) $F_{e}(v)$ vs. $v_{\perp} / v_{\text {th }}$ and $v_{\|} / v_{\text {th }}$, for four different time steps corresponding to $\omega_{p e} t=500$, 1000,1500 , and 2000. The top four panels correspond to the WT simulation, while the bottom four panels show results from the PIC code simulation. 
corresponding to $\omega_{p e} t=500$ and 1000. For $\omega_{p e} t=1500$ and 2000, it is evident that the WT calculation exaggerates the velocity space diffusion of the beam. For the PIC code simulation, the beam is spread along $v_{\perp}$ as in case 2, but the peak velocity portion of the beam along $v_{\|}$does not evolve much. It is interesting to note that for $\omega_{p e} t=2000$, the PIC simulation indicates parallel acceleration of electrons in both positive and negative portions of $v_{\|}$axis. This feature is absent in the WT calculation. In an overall sense, while there exist some discrepancies, the qualitative agreement is arguably present, especially for relatively early times. This assessment notwithstanding, the present case 3 study implies that the beam speed of $V_{b} / v_{\text {th }}=10$ may be at the limit of validity for WT theory, at least from the standpoint of electron VDF.

However, as for the Langmuir turbulence, the agreement between the WT method and the PIC code simulation is not that bad. Indeed, as Figure 8 demonstrates, the Langmuir turbulence spectrum and the simulated longitudinal electric field spectrum are qualitatively similar. The overall morphologies of the 2D spectra for both methods somehow produce rather consistent results, including the fact that even the WT simulation generates intense Langmuir condensation, which was missing in the previous two cases.

Finally, moving on to the radiation emission pattern, and jumping to the final state corresponding to $\omega_{p e} t=2000$, which is shown in the top and bottom panels of Figure 9, one may immediately appreciate the similarities in the WT versus PIC simulated radiation pattern, which show fundamental/secondharmonic emissions, as well as weak third-harmonic emission. Even in the PIC code result, numerical noise notwithstanding, the third-harmonic emission is easy to identify. Now, as for relatively early time periods, especially for $\omega_{p e} t=500$ and 1000 , the PIC code simulation is still too noisy to visually identify and discern clear radiation emission at the harmonics. This contrasts with the WT calculation, which is free of noise problems. By the time the PIC code simulation is carried out to $\omega_{p e} t=1500$, however, the radiation emission pattern begins to manifest itself, albeit rather faintly.

In Ziebell et al. (2015), the authors analyzed the detailed physics of the plasma emission. Specifically, they discussed that the fundamental emission takes place as a result of combined processes of $L$ mode decaying into $T$ and $S$ modes, as well as the scattering involving the beating of $L$ and $T$ modes mediated by the particles. They mentioned that both the decay and scattering mechanisms are governed by a coupling coefficient of the form

$$
\frac{\left(\boldsymbol{k} \times \boldsymbol{k}^{\prime}\right)^{2}}{k^{2} k^{\prime 2}} \propto \sin ^{2} \vartheta,
$$

where $\vartheta$ represents the angle between the two vectors $\boldsymbol{k}^{\prime}$ and $\boldsymbol{k}$. They also argued that the fundamental emission along the direction specified by $\vartheta=0$ should be prohibited, thus resulting in the dipole radiation. In the numerical simulation, whether it is based upon the WT theory, or it is by means of the direct PIC code method, the dipolar pattern associated with the fundamental radiation is difficult to discern, because it involves a narrow region around $k \sim 0$.

For the second-harmonic emission, on the other hand, it is well known that the fundamental emission mechanism involves the coalescence of two oppositely traveling Langmuir waves with the coupling coefficient of the form

$$
\frac{\left(\boldsymbol{k} \times \boldsymbol{k}^{\prime}\right)^{2}}{k^{2} k^{\prime 2}}\left(k^{\prime 2}-\left|\boldsymbol{k}-\boldsymbol{k}^{\prime}\right|^{2}\right)^{2} \sim \sin ^{2} \vartheta \cos ^{2} \vartheta .
$$

This implies a quadrupole pattern, but because the radiation emission generally involves multiple wave modes, the above coupling coefficient is to be integrated over $\boldsymbol{k}^{\prime}$, or equivalently, $\vartheta$, hence, the strict quadrupole emission is not evident in reality.

Ziebell et al. (2015) also confirmed the earlier theories of third-harmonic and higher emission (Zheleznyakov \& Zlotnik 1974; Cairns 1987b; Kliem et al. 1992). The coupling coefficient for the higher harmonic emission is given by

$$
\left(1+\frac{\left(\boldsymbol{k} \cdot \boldsymbol{k}^{\prime}\right)^{2}}{k^{2} k^{\prime 2}}\right) \sim 1+\cos ^{2} \vartheta
$$

Ziebell et al. (2015) also reminded readers that the thirdharmonic plasma emission associated with the solar radio bursts is quite rare (Takakura \& Yousef 1974; Zlotnik et al. 1998; Brazhenko et al. 2012).

Finally, Ziebell et al. (2015) analyzed the details of the various emission mechanisms by artificially turning certain terms in the $T$ wave kinetic equation on or off in order to investigate the consequences of doing so. By employing such an approach, they confirmed the various theories that have been proposed in the literature concerning the radiation emission mechanisms. Their conclusion still holds, and it is thus unnecessary to repeat their analysis. It should be noted, however, that this methodology is unique to theoretical approaches such as the WT methodology, since with the PIC code simulation, despite all the rigor inherent to the approach, it is difficult to distinguish the underlying individual physical processes, since all of them operate simultaneously. This shows that the WT and PIC simulation tools mutually complement each other, and when employed judiciously, may constitute a powerful research tool for the study of solar radio bursts.

\section{Summary and Conclusions}

The purpose of the present paper has been to investigate the plasma emission process by making use of two different approaches, and to discuss the compatibility between the results obtained from these two approaches. The plasma emission is generally acknowledged to be the fundamental radiation emission mechanism for solar type II and type III radio bursts phenomena.

In one of the approaches, we have utilized a self-consistent system of coupled equations, obtained using the framework of WT theory, in order to study the time evolution of the VDF of the electrons and of the spectra of electrostatic and transverse waves. The formulation incorporates the effects of different physical mechanisms. In such an approach the roles of different physical processes can be identified unambiguously by turning certain terms on and off, which has in fact been done by Ziebell et al. (2015). The WT formulation therefore is a convenient tool to test the validity of various theories proposed in the literature for the generation of plasma emission. The complete set of equations can also be solved without a priori assumptions in order to quantitatively analyze the plasma emission process, as was done by Ziebell et al. (2015). On the other hand, the WT theory, as with any analytical theory, is based upon a series of 

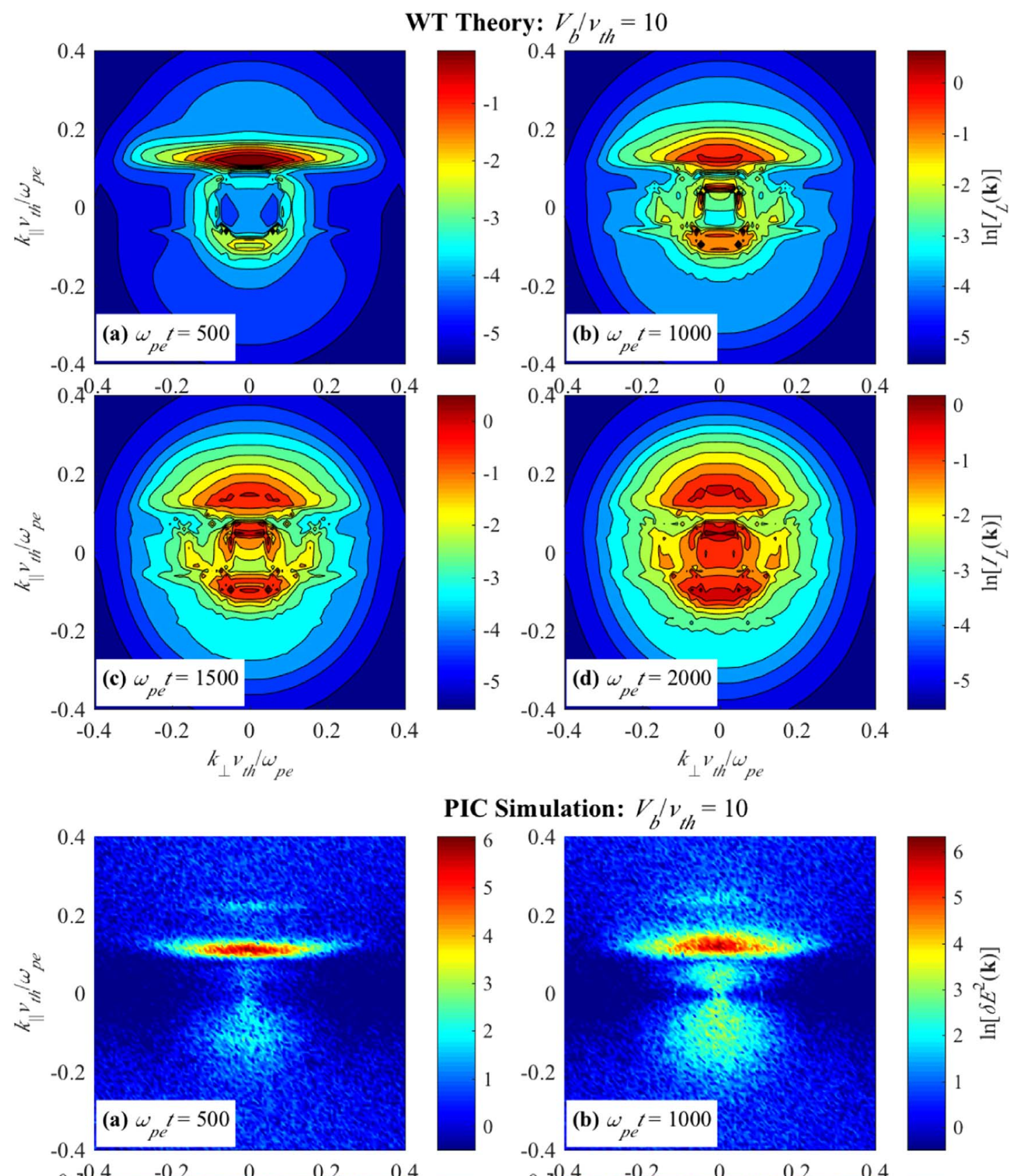

PIC Simulation: $V_{b} / v_{t h}=10$
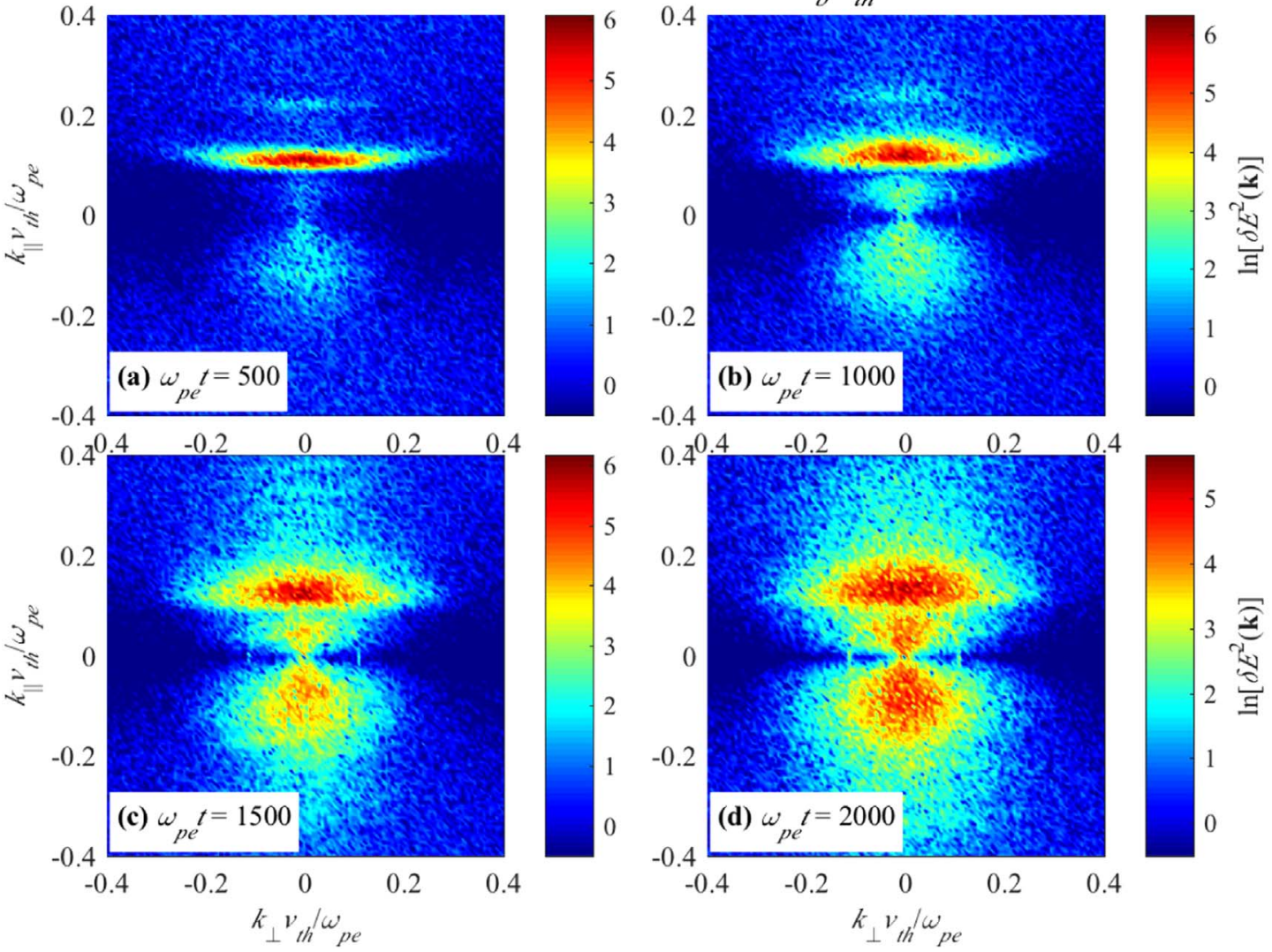

Figure 8. Case $3\left(V_{b} / v_{\text {th }}={ }^{\prime} 0\right)$ : Langmuir wave spectral intensity $I_{L}(\boldsymbol{k})$, in the case of the WT simulation (top four panels), and total electric field intensity $\delta E^{2}(\boldsymbol{k})$, in the case of the PIC code simulation (bottom four panels), vs. $k_{x} v_{\text {th }} / \omega_{p e}$ and $k_{z} v_{\text {th }} / \omega_{p e}$, for $\omega_{p e} t=500,1000,1500$, and 2000. 
WT Theory: $V_{b} / v_{\text {th }}=10$
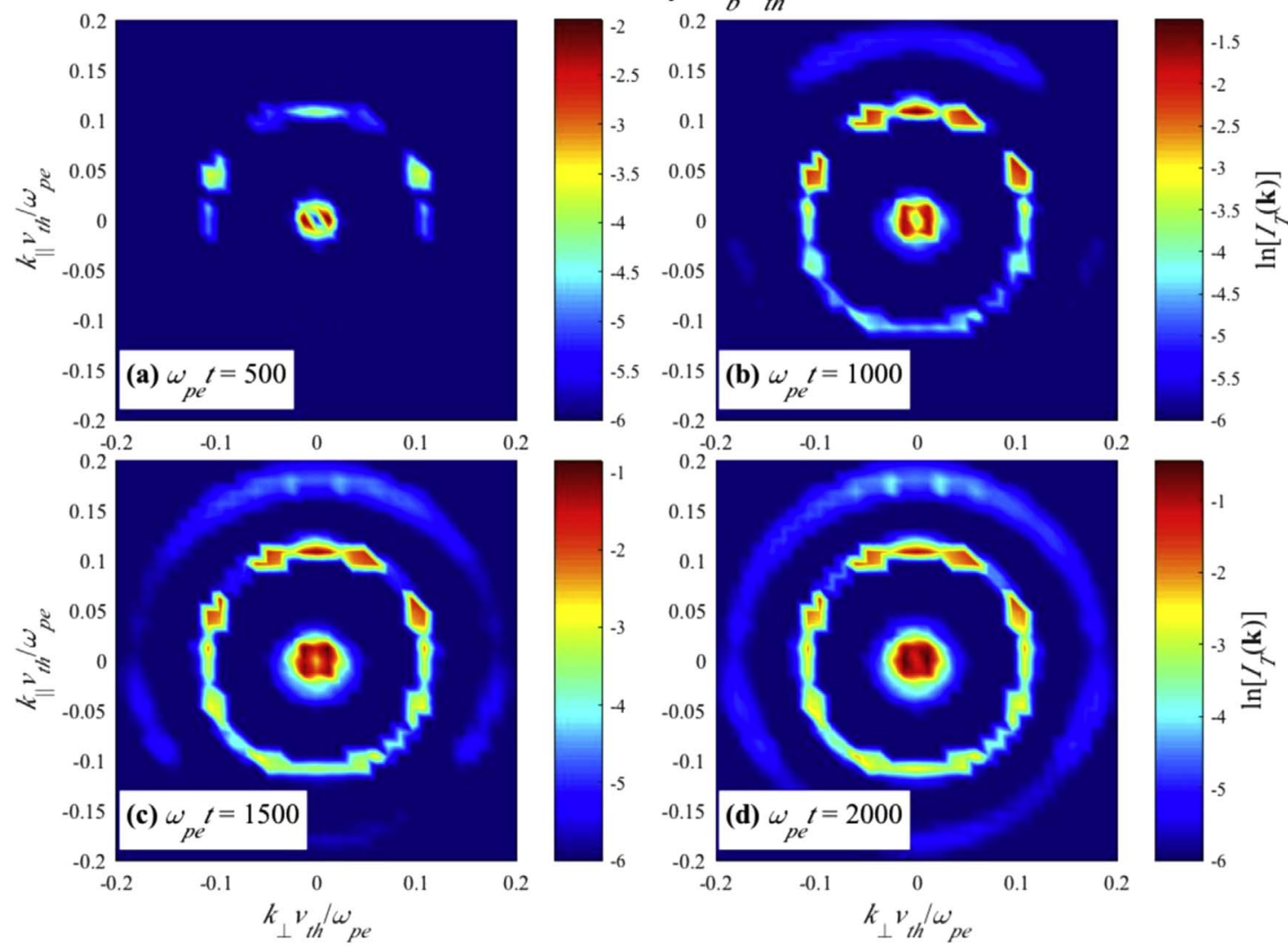

PIC Simulation: $V_{b} / v_{t h}=10$
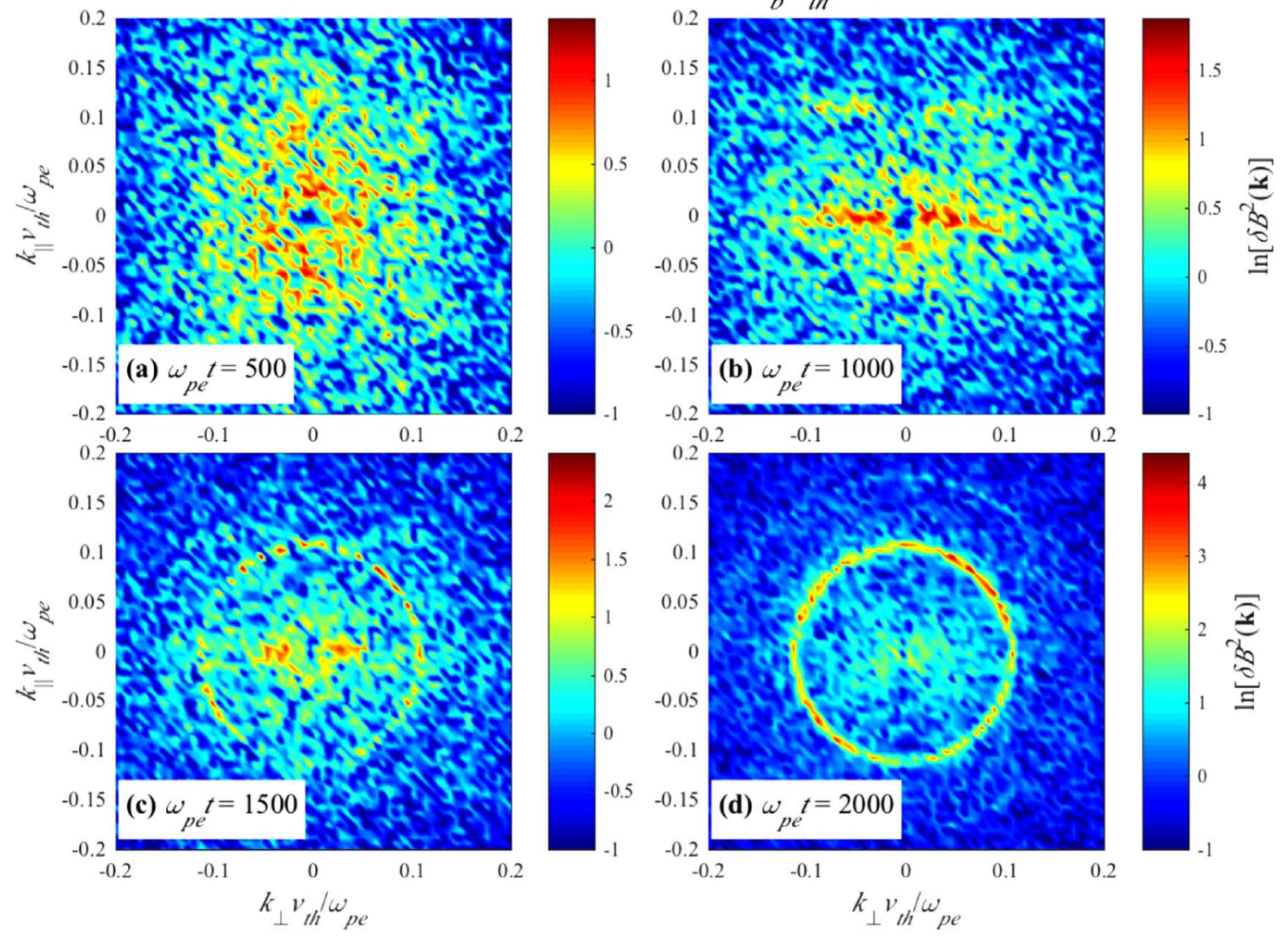

Figure 9. Case $3\left(V_{b} / v_{\mathrm{th}}=10\right)$ : radiation spectral intensity $I_{T}(\boldsymbol{k})$, in the case of the WT simulation (top four panels), and total magnetic field intensity $\delta B^{2}(\boldsymbol{k})$, in the case of the PIC code simulation (bottom four panels), vs. $k_{x} v_{\text {th }} / \omega_{p e}$ and $k_{z} v_{\text {th }} / \omega_{p e}$, for $\omega_{p e} t=500,1000,1500$, and 2000. 
assumptions whose limits have not been clearly defined or tested.

The second approach employed in the present investigation has been the direct numerical simulation, based upon the PIC paradigm. The PIC simulations rely on a smaller number of theoretical constraints than the approach based on WT theory, as such an approach basically solves the Lorentz equations of motion for a collection of charged particles, plus the Maxwell equations. Nonetheless, such a numerical approach is not without some shortcomings. For instance, the necessity of discretization and the finite grid size in velocity space and in wave number space, place some limitations on the resolutions of very small wavelengths. The simulation method also needs to take into account a large number of particles inside a cell in order to reduce the numerical noise, with a consequent burden on the requirements for computational resources. In addition, it is not so easy to create clear diagnostics for the phenomena that occur in the system, as all the mechanisms act simultaneously, and cannot be arbitrarily turned on or off for verification of certain physical processes that operate in the system. In contrast, the WT approach naturally lends itself to such manipulations. The WT method requires far less computational resources.

We have made use of these two approaches to study a plasma system containing one ion population and two electron populations, constituted by an initially Maxwellian background and a tenuous beam. We have considered the same parameters that were utilized in a previous paper in which the WT equations were employed in order to discuss in detail the physical mechanisms involved in the plasma emission process (Ziebell et al. 2015). The WT analysis of the present paper is essentially the same as that of Ziebell et al. (2015), except we have considered a wider velocity space in order to minimize the boundary effects. The initial setup for the PIC code simulation is consistent with that of the WT analysis by Ziebell et al. (2015). Of course, the PIC code assumes additional parameters, such as the grid size and the number of particles per cell, etc., but the physical conditions are consistent with those of Ziebell et al. (2015).

The numerical results discussed in detail indicate that the results obtained with the WT approach and with the PIC simulations are largely compatible. Regarding the evolution of the electron distribution function, both approaches show the formation of a plateau in the beam region, within compatible timescales. The agreement between the WT and the PIC results is more noticeable for the case of lower beam velocity, which is not too surprising, since the WT theory is based upon the assumption of weak wave growth and low wave energy density when compared to the particle thermal energy density. For the intermediate and high beam velocity cases considered in the present paper, we noticed that after the formation of the plateau, the high-velocity part of the beam in the WT results is slightly wider along the $v_{\perp}$ direction than that in the PIC results. Moreover, the high-velocity case (specifically for $\left.V_{b} / v_{\text {th }}=10\right)$ WT calculation resulted in the formation of an extended tail along the forward direction, which is not seen in the PIC simulation results. This appears to be an indication that the high beam velocity case of $V_{b} / v_{\text {th }}=10$, when all other parameters are held constant, corresponds to the limit of applicability of the WT equation.

Regarding the spectra of waves, the WT approach singles out spectra for each eigenmode, which is built into the theory. In contrast, for the PIC simulation, the various eigenmodes, such as Langmuir or transverse waves, must be carefully interpreted. For instance, in order to construct the spectrum of Langmuir waves on the basis of the PIC simulation, we have considered only the electric field component along the direction parallel to the beam velocity. This may separate electrostatic waves from transverse waves, but may still retain some amount of intensity associated with the nonlinear harmonics of electrostatic waves, hence the interpretation of the PIC simulation result, as far as Langmuir waves are concerned, is not without some ambiguities. Nevertheless, the results obtained with the two approaches are largely compatible. Both the WT results and the PIC results show the formation of the primary Langmuir wave peak, with comparable widths along the directions of parallel and perpendicular wave numbers, the growth of backward propagating waves, which are also characterized by consistent widths in wave number space, and also the spread of the primary peak toward the region of smaller wave numbers. A discrepancy remains, mostly in the cases of low and intermediate beam velocities, namely, $V_{b} / v_{\text {th }}=6$ and 8 , in that whereas the PIC simulation shows the early appearance of waves for $k \simeq 0$, the so-called Langmuir condensation effect, in the WT results the region of very small values of $k$ is not quite attained, until the final computational time attained in our analysis. In the case of higher beam velocity which has been considered, namely, $V_{b} / v_{\text {th }}=10$, on the other hand, the WT results also show some growth of waves at $k \simeq 0$. The reason for this localized discrepancy between the two approaches is not yet completely understood. In an overall sense, however, the agreement between the WT and PIC methods is more consistent than the electron velocity distribution, which is interesting.

In order to obtain information about the spectra of electromagnetic waves in the case of the PIC simulation results, we have taken into account the total magnetic field intensity. The spectrum of the magnetic field fluctuations is then used for comparison with the spectrum of transverse waves computed on the basis of the WT method. The comparative analysis produced largely favorable results, although because of numerical noise associated with the PIC code, and because of the generally low level of radiation, the relatively early time results do not clearly show easily identifiable plasma emission. The compatibility between the WT and PIC results was seen to improve with the increase of beam velocity. Clear demonstration of the radiation emission was difficult to show with the limited numerical setup adopted in the PIC code. This implies that the simulation of plasma emission requires a large number of particles per cell in order to reduce the numerical noise, which requires high computational resources.

To conclude, the PIC code simulation is supposed to be more rigorous, but it necessitates computationally intense efforts. In contrast, while the WT theory is a reduced approach, the comparative analysis presented here suggests that the use of WT theory can be reliable, if it is carefully applied to a parameter regime for which the theory is valid. The present investigation has focused on three examples, but a more systematic statistical survey of the parameter space could be carried out in order to further establish the region of validity of the WT approach. The present paper also indicates the possibility of improved agreement between the WT approach and the PIC simulation approach if the numerical noise can be 
reduced in the PIC simulations. In short, we find that both the WT theory and PIC code simulations are useful research tools in the fundamental study of solar radio bursts problem, as they are mutually complementary.

The present research was supported in part by the BK21 plus grant from the National Research Foundation (NRF) of the Republic of Korea, to Kyung Hee University. L.F.Z. and R.G. acknowledge support provided by Conselho Nacional de Desenvolvimento Científico e Tecnológico (CNPq), grants No. 304363/2014-6 and 307626/2015-6. This study was also financed in part by the Coordenação de Aperfeiçoamento de Pessoal de Nível Superior-Brasil (CAPES)_Finance Code 001. P.H.Y. acknowledges the grant from the GFT Charity, Inc., to the University of Maryland. This work was partly carried out while P.H.Y. was visiting the Korea Astronomy and Space Science Institute (KASI). E.L. acknowledges support by Space Core Technology Development Program through the NRF of Korea funded by the Ministry of Science and ICT (NRF-2017M1A3A3A02016781).

\section{ORCID iDs}

Sang-Yun Lee (iD https://orcid.org/0000-0003-3177-5725

L. F. Ziebell (iD https://orcid.org/0000-0003-0279-0280

P. H. Yoon (iD https://orcid.org/0000-0001-8134-3790

R. Gaelzer (ib https://orcid.org/0000-0001-5851-7959

\section{References}

Bastian, T. S., Benz, A. O., \& Gary, D. E. 1998, ARA\&A, 36, 131 Benz, A. O. 2017, LRSP, 14, 2

Brazhenko, A. I., Melnik, V. N., Konovalenko, A. A., et al. 2012, OAP, 25,181

Cairns, I. H. 1987a, JPIPh, 38, 169

Cairns, I. H. 1987b, JP1Ph, 38, 199

Cane, H. V., Erickson, W. C., \& Prestage, N. P. 2002, JGR, 107, 1315

Ergun, R. E., Larson, D., Lin, R. P., et al. 1998, ApJ, 503, 435

Gaelzer, R., Ziebell, L. F., Viñas, A. F., Yoon, P. H., \& Ryu, C. M. 2008, ApJ, 677,676

Ganse, U., Kilian, P., Spanier, F., \& Vainio, R. 2012a, ApJ, 751, 145

Ganse, U., Kilian, P., Vainio, R., \& Spanier, F. 2012b, SoPh, 280, 551

Ginzburg, V. L., \& Zheleznyakov, V. V. 1958, SvPhA, 2, 653

Goldman, M. V. 1983, SoPh, 89, 403

Goldman, M. V., \& DuBois, D. F. 1982, PhFl, 25, 1062

Gosling, J. T., Skoug, R. M., \& McComas, D. J. 2003, GeoRL, 30, 1697

Henri, P., Sgattoni, A., Briand, C., Amiranoff, F., \& Riconda, C. 2019, JGR, in press

Hudson, H. 2011, SSRv, 158, 5

Kaplan, S. A., \& Tsytovich, V. N. 1968, SvPhA, 11, 956

Karlický, M., \& Vandas, M. 2007, P\&SS, 55, 2336
Kasaba, Y., Matsumoto, H., \& Omura, Y. 2001, JGR, 106, 18693

Kliem, B., Krueger, A., \& Treumann, R. A. 1992, SoPh, 140, 149

Kontar, E. P. 2001a, PPCF, 43, 589

Kontar, E. P. 2001b, SoPh, 202, 131

Kontar, E. P. 2001c, A\&A, 375, 629

Krafft, C., Volokitin, A. S., \& Krasnoselskikh, V. V. 2013, ApJ, 778, 111

Li, B., Cairns, I. H., \& Robinson, P. A. 2008, JGR, 113, A06104

Li, B., Cairns, I. H., \& Robinson, P. A. 2011, ApJ, 730, 20

Li, B., Cairns, I. H., \& Robinson, P. A. 2012, SoPh, 279, 173

Li, B., Robinson, P. A., \& Cairns, I. H. 2006, PhPl, 13, 092902

Li, B., Willes, A. J., Robinson, P. A., \& Cairns, I. H. 2005a, PhPl, 12, 012103

Li, B., Willes, A. J., Robinson, P. A., \& Cairns, I. H. 2005b, PhPl, 12, 052324

López, R. A., \& Yoon, P. H. 2018, JGR, 123, 8924

Melrose, D. B. 1982, AuJPh, 35, 67

Melrose, D. B. 1987, SoPh, 111, 89

Ratcliffe, H., Bian, N. H., \& Kontar, E. P. 2012, ApJ, 761, 176

Ratcliffe, H., Brady, C. S., Che Rozenan, M. B., \& Nakariakov, V. M. 2014, $\mathrm{PhPl}, 21,122104$

Reid, H. A. S., \& Kontar, E. P. 2017, A\&A, 598, A44

Reid, H. A. S., \& Ratcliffe, H. 2014, RAA, 14, 773

Reiner, M. J., Goetz, K., Fainberg, J., et al. 2009, SoPh, 259, 255

Rha, K., Ryu, C.-M., \& Yoon, P. H. 2013, ApJL, 775, L21

Rhee, T., Ryu, C.-M., Woo, M., et al. 2009a, ApJ, 694, 618

Rhee, T., Woo, M., \& Ryu, C.-M. 2009b, JKPS, 54, 313

Robinson, P. A., \& Cairns, I. H. 1998a, SoPh, 181, 363

Robinson, P. A., \& Cairns, I. H. 1998b, SoPh, 181, 395

Takakura, T., \& Yousef, S. 1974, SoPh, 36, 451

Thurgood, J. O., \& Tsiklauri, D. 2015, A\&A, 584, A83

Tsytovich, V. N. 1967, SvPhU, 9, 805

Umeda, T. 2010, JGR, 115, A01204

Wild, J. P. 1950a, AuSRA, 3, 399

Wild, J. P. 1950b, AuSRA, 3, 541

Wild, J. P., \& McCready, L. L. 1950, AuSRA, 3, 387

Wild, J. P., Murray, J. D., \& Rowe, W. C. 1954, AuJPh, 7, 439

Yoon, P. H. 2000, PhPl, 7, 4858

Yoon, P. H. 2005, PhPl, 12, 042306

Yoon, P. H. 2006, PhPl, 13, 022302

Yoon, P. H., Hong, J., Kim, S., et al. 2012a, ApJ, 755, 112

Yoon, P. H., Ziebell, L. F., Gaelzer, R., \& Pavan, J. 2012b, PhPl, 19, 102303

Zheleznyakov, V. V., \& Zlotnik, E. Y. 1974, SoPh, 36, 443

Ziebell, L. F., Gaelzer, R., Pavan, J., \& Yoon, P. H. 2008a, PPCF, 50, 085011

Ziebell, L. F., Gaelzer, R., \& Yoon, P. H. 2001, PhPl, 8, 3982

Ziebell, L. F., Gaelzer, R., \& Yoon, P. H. 2008b, PhPl, 15, 032303

Ziebell, L. F., Petruzzellis, L. T., Yoon, P. H., Gaelzer, R., \& Pavan, J. 2016, ApJ, 818, 61

Ziebell, L. F., Yoon, P. H., Gaelzer, R., \& Pavan, J. 2012, PPCF, 54, 055012

Ziebell, L. F., Yoon, P. H., Gaelzer, R., \& Pavan, J. 2014a, PhPl, 21, 012306

Ziebell, L. F., Yoon, P. H., Gaelzer, R., \& Pavan, J. 2014b, ApJL, 795, L32

Ziebell, L. F., Yoon, P. H., Pavan, J., \& Gaelzer, R. 2011a, ApJ, 727, 16

Ziebell, L. F., Yoon, P. H., Pavan, J., \& Gaelzer, R. 2011b, PPCF, 53, 085004

Ziebell, L. F., Yoon, P. H., Petruzzellis, L. T., Gaelzer, R., \& Pavan, J. 2015, ApJ, 806, 237

Ziebell, L. F., Yoon, P. H., Simões, F. J. R., Gaelzer, R., \& Pavan, J. 2014c, $\mathrm{PhPl}, 21,010701$

Zlotnik, E. Y., Klassen, A., Klein, K. L., Aurass, H., \& Mann, G. 1998, A\&A, 331,1087 